\title{
First Steps toward Formal Controller Synthesis for Bipedal Robots with Experimental Implementation
}

\author{
Aaron D. Ames ${ }^{\mathrm{a}}$, Paulo Tabuada ${ }^{\mathrm{b}}$, Austin Jones ${ }^{\mathrm{a}}$, Wen-Loong Ma ${ }^{\mathrm{a}}$, Matthias \\ Rungger $^{c}$, Bastian Schürmann ${ }^{c}$, Shishir Kolathaya ${ }^{a}$, Jessy W. Grizzle ${ }^{\mathrm{d}}$ \\ ${ }^{a}$ Georgia Institute of Technology \\ ${ }^{b}$ University of California, Los Angeles \\ ${ }^{c} T U$ München \\ ${ }^{d}$ University of Michigan
}

\begin{abstract}
Bipedal robots are prime examples of complex cyber-physical systems (CPS). They exhibit many of the features that make the design and verification of CPS so difficult: hybrid dynamics, large continuous dynamics in each mode (e.g., 10 or more state variables), and nontrivial specifications involving nonlinear constraints on the state variables. In this paper, we propose a two-step approach to formally synthesize controllers for bipedal robots so as to enforce specifications by design and thereby generate physically realizable stable walking. In the first step, we design outputs and classical controllers driving these outputs to zero. The resulting controlled system evolves on a lower dimensional manifold and is described by the hybrid zero dynamics governing the remaining degrees of freedom. In the second step, we construct an abstraction of the hybrid zero dynamics that is used to synthesize a controller enforcing the desired specifications to be satisfied on the full order model. Our two step approach is a systematic way to mitigate the curse of dimensionality that hampers the applicability of formal synthesis techniques to complex CPS. Our results are illustrated with simulations showing how the synthesized controller enforces all the desired specifications and offers improved performance with respect to a classical controller. The practical relevance of the results is illustrated experimentally on the bipedal robot AMBER 3.
\end{abstract}

Keywords: Hybrid Systems, Formal Methods, Nonlinear Control, Bipedal Robotics

Email addresses: ames@gatech.edu (Aaron D. Ames), tabuada@ee.ucla.edu (Paulo Tabuada), austinjones@gatech.edu (Austin Jones), wenlongma@gatech.edu (Wen-Loong Ma), matthias.rungger@tum.de (Matthias Rungger), bastian.schuermann@in.tum.de (Bastian Schürmann), shishirny@gatech.edu (Shishir Kolathaya), grizzle@eecs.umich.edu (Jessy W. Grizzle) 

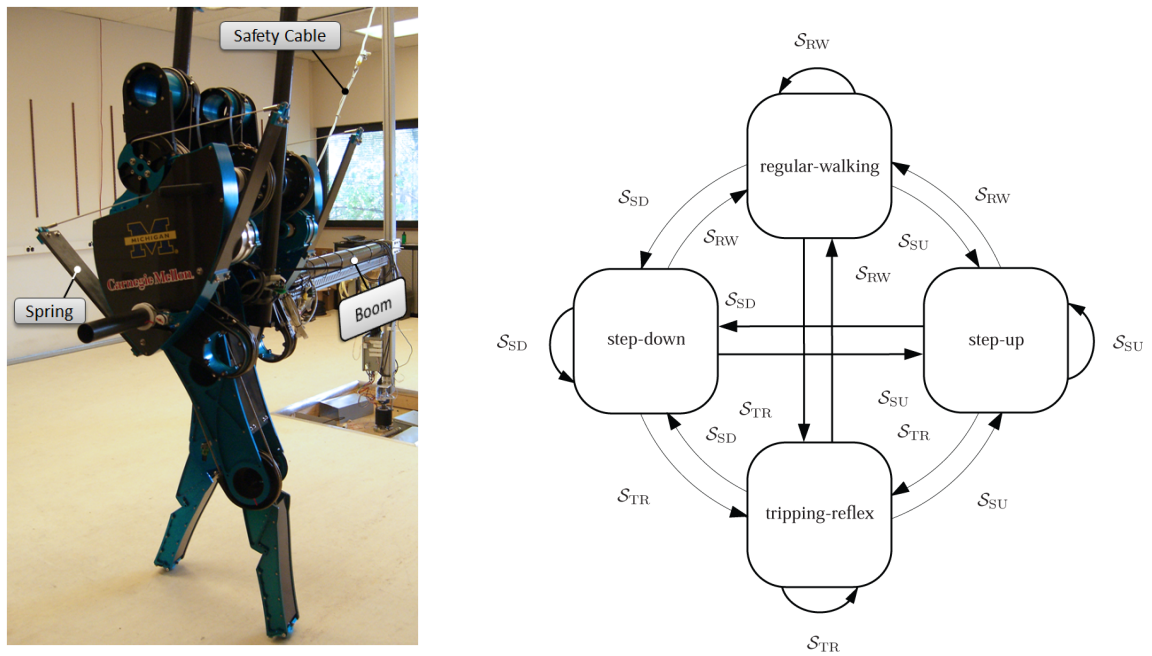

Figure 1: The planar (2D) biped MABEL was developed for the study of dynamic locomotion. High-level motion primitives were used on MABEL to allow walking over rough ground without tripping. While tools for automatic low-level control algorithm synthesis are well developed, at the state machine level, all tuning was done by hand for lack of appropriate tools. "Probable correctness" was established through extensive simulation and experiments.

\section{Introduction}

Legged robots are complex dynamic cyber-physical system (CPS). As a concrete example, consider MABEL shown in Fig. 1. This bipedal robot possesses nonlinear, non-order preserving, non-convex dynamics described by a hybrid model with 14 state variables and four actuators [26]. To enable MABEL to accept a set of high-level locomotion commands over a network, and successfully execute the commands while responding automatically and safely to uncertainty in the assumed profile of the environment, the finite state machine shown in Fig. 1 was designed [25]. It allowed MABEL to compose on the fly a set of low-level control algorithms executing a handful of motion primitives. A team of graduate students hand-tuned the transition conditions among the various nodes of the state machine. Each time a small change was made in one of the software or hardware components, such as adjusting a transition condition or adding a sensor, the entire state machine had to be completely retested, leading often to the redesign of other software components. There is a pressing need to understand this, and more general CPSs, in a way that allows for the automatic synthesis of embedded control software that is provably correct by construction.

In this paper, we begin to lay the groundwork for this correct-by-construction control software design process in the context of dynamic systems. In particular, the specific bipedal robot that will be studied is AMBER 3 as shown in Fig. 2. We consider a walking gait with the simplest discrete structure, resulting in a single-mode hybrid model with 12 state variables and 6 actuators. While we seek formal guarantees on the behavior of the 12-dimensional closed- 

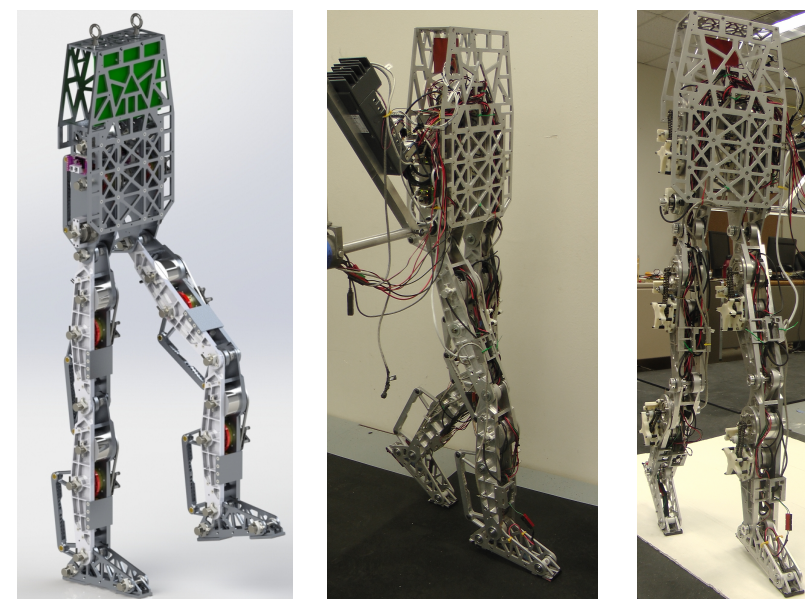

Figure 2: Bipedal walking robot AMBER 3.

loop system, we do not propose to perform formal synthesis on a model this large. Similarly to the work in $[8,9]$, we focus on the regulation of a subset of system states and use advanced nonlinear control methods to transform the complex dynamics to a simpler, more tractable system which is amenable to the correct-by-construction synthesis techniques. In contrast to $[8,9]$, where the authors exploit differential flatness to reduce the nonlinear synthesis problem to a controller design problem for a chain of integrators, our method applies to the aforementioned hybrid system with non-flat outputs. Specifically, in our approach the chain of integrators is forced to be at equilibrium and we apply the symbolic abstraction techniques to a hybrid system that lives on an attractive, hybrid-invariant, low-dimensional manifold which is "complementary" to the state space of the integrators $[2,35]$. The low-dimensional hybrid subsystem is called the Hybrid Zero Dynamics (HZD), and its solutions can be used to reconstruct the solutions of the high-dimensional hybrid system. The end result is the ability to guarantee specifications on the full-order high-dimensional system via the reduced order representation encoded by the HZD.

There is a growing interest in the synthesis of correct-by-construction controllers for robotic applications as evidenced by the growing body of work on this topic $[32,10,19,11]$. Although the techniques we employ for synthesis are based on the symbolic abstraction techniques described in [31], what sets the results in this paper apart from prior work is the complexity of the system being controlled. In particular, as previously mentioned, the hybrid model for AMBER 3 requires 12 state variables, which is larger than any system previously reported in the literature for which correct-by-construction control has been synthesized. The key to scaling the symbolic controller synthesis techniques to this level of complexity is the new design flow based on the HZD. This is the main contribution of the paper as we believe that its applicability transcends 
the specific formal synthesis technique we employ and the robotics domain in which we develop the result.

The results in this paper are based on previous work by the authors on two lines of research that have been independently pursued in the past: 1) control of bipedal robots via hybrid zero dynamics and 2) synthesis of controllers via finite-state abstractions. In order to combine techniques from these two different areas several new results, formalized in Theorems 1 through 4 , had to be proved. Theorems 1 and 2 are new because they represent a notion of physically realizable walking that had not been treated previously in the context of hybrid zero dynamics; specifically, previous work focused on asymptotic stability of periodic trajectories lying in the zero dynamics manifold, while for the present work, a more general notion of aperiodic upright walking gaits is required. Theorem 3 is new since prior work by the authors on the construction of abstractions for hybrid systems considered only switched systems. The hybrid model considered in this paper, as presented in Sect. 2, is not a switched system since it is equipped with a nontrivial guard and reset map

This paper is an extension of the conference paper [5]. In addition to new simulation results, this paper features the first experimental implementation of the proposed methodology of combining HZD with formal methods. Initial results indicate that the controller based on formal methods enforces constraints better than a controller based only on regulating system outputs. The platform we consider in this paper, AMBER 3, is a successor to AMBER 2 [21,38], the platform modeled in [5]. AMBER 3 is approximately $40 \%$ larger than AMBER 2 and its size more closely approximates an adult human. The inclusion of a full-size torso also enables better balancing while walking. Finally, the motors used on AMBER 3 are approximately ten times more powerful than the ones used on AMBER 2

The remainder of the paper is organized as follows: We begin by introducing hybrid systems in Sect. 2, with a special focus on the hybrid model of walking robots with a single domain. In addition, we introduce the notion of a stable walking gait (without requiring periodicity), and give conditions that ensure that these walking gaits are physically realizable. In Sect. 3 , we introduce a means for dimension reduction through the use of a pre-feedback controller that renders a low-dimensional surface hybrid invariant yielding HZD. Importantly, for this initial result, the controller also results in linear dynamics on the hybrid zero dynamics surface and, ultimately, a hybrid system with two-dimensional continuous linear dynamics. We establish the first theoretic results of the paper in Sect. 3, wherein it is shown that solutions of the HZD lift to solutions of the full-order hybrid system and that the HZD manifold is stable as a set. Section 4 introduces the main results of the paper: a means for formally constructing controllers via the HZD to yield provably stable walking gaits that satisfy physical realizability constraints. The paper concludes with Sect. 5, where simulation results and experiments on AMBER 3 using correct-by-construction control are presented. 


\section{Bipedal Robot Models, Walking Gaits and Physical Constraints}

In this section, we present a formalism for hybrid systems that is sufficient for modeling bipedal walking robots. After the introduction of these models, we present a definition of a walking gait for a bipedal robot along with associated physical realizability constraints. This will set the stage for formal controller synthesis for bipedal robots.

Hybrid Systems \& Executions. We begin by introducing hybrid (control) systems (also referred to as systems with impulse effects $[13,14]$ ). We consider hybrid systems with one domain because the specific biped models considered in this paper applies to flat-footed walking; for more complex foot behavior, more elaborate hybrid systems must be considered [6, 12, 14, 15, 20, 38].

A hybrid control system is a tuple,

$$
\mathscr{H} \mathscr{C}=(\mathcal{D}, U, S, \Delta, f, g),
$$

where $\mathcal{D}$ is the domain with $\mathcal{D} \subseteq \mathbb{R}^{n}$ a smooth submanifold of the state space $\mathbb{R}^{n}$, $U \subseteq \mathbb{R}^{m}$ is the set of admissible controls, $S \subset \mathcal{D}$ is a proper subset of $\mathcal{D}$ called the guard or switching surface, $\Delta: S \rightarrow \mathcal{D}$ is a smooth map called the reset map, and $(f, g)$ is a control system on $\mathcal{D}$, i.e., in coordinates: $\dot{x}=f(x)+g(x) u$ with $u \in U$. A hybrid system is a hybrid control system with $U=\{0\}$; a particular example would include a closed-loop hybrid system, meaning that a feedback controller has been applied, defining the inputs as functions of the state. In this case,

$$
\mathscr{H}=(\mathcal{D}, S, \Delta, f),
$$

where $f$ is a dynamical system on $\mathcal{D} \subseteq \mathbb{R}^{n}$, i.e., $\dot{x}=f(x)$.

For the sake of simplicity, and without loss of generality for the formal results presented, we will consider infinite solutions (or hybrid flows or executions) of a hybrid system $\mathscr{H}$. Motivated by existing definitions [20, 35, 12, 18], we define a solution to a hybrid system $\mathscr{H}$ by the tuple:

$$
\chi^{\mathscr{H}}=(\mathcal{I}, \mathcal{C}),
$$

where $\mathcal{I}=\left\{I_{i}\right\}_{i \in \mathbb{N}}$ is a hybrid interval where $I_{i}=\left[\tau_{i}, \tau_{i+1}\right]$ with $\tau_{i}, \tau_{i+1} \in \mathbb{R}$ and $\tau_{i} \leq \tau_{i+1}$, and $\mathcal{C}=\left\{c_{i}\right\}_{i \in \mathbb{N}}$ is a collection of solutions to $f$, i.e., $\dot{c}_{i}(t)=f\left(c_{i}(t)\right)$ for all $t \in I_{i}$. In addition, we require the following conditions to hold:

(i) $c_{i}(t) \in \mathcal{D}$ for all $t \in I_{i}, i \in \mathbb{N}$,

(ii) $\tau_{i+1}=\inf \left\{t \geq \tau_{i}: c_{i}(t) \in S\right\}$,

(iii) $\Delta\left(c_{i}\left(\tau_{i+1}\right)\right)=c_{i+1}\left(\tau_{i+1}\right)$.

The initial condition for a hybrid flow is $x_{0}=c_{0}\left(\tau_{0}\right)$. When we wish to make explicit the initial condition of $\chi^{\mathscr{H}}$ we will write $\chi^{\mathscr{H}}\left(x_{0}\right)$. 
Robotic Hybrid System Models. Utilizing the formulation of hybrid systems, we will now construct hybrid system models for bipedal robots. Specifically, we will consider a hybrid control system of the form:

$$
\mathscr{H} \mathscr{C}_{R}=\left(\mathcal{D}_{R}, U_{R}, S_{R}, \Delta_{R}, f_{R}, g_{R}\right) .
$$

The constructions of this section will be presented in the general case of a robot with a single discrete phase of walking, i.e., they will not be specific to the robot-AMBER 3- that will be considered in this paper. As a result they are applicable to both 2D and 3D robots in the case of full actuation, including humanoid robots. It is important to note that the constructions considered in this paper do not apply to robots with underactuation (since, in this case, there will not be actuation in the zero dynamics), yet future work will be devoted to considering this case as well.

Continuous Dynamics: Let $\mathcal{Q}_{R}$ be the configuration space of a robot with $n$ degrees of freedom, i.e., $n=\operatorname{dim}\left(\mathcal{Q}_{R}\right)$, with coordinates $\theta \in \mathcal{Q}_{R}$. For the sake of definiteness, it may be necessary to choose $\mathcal{Q}_{R}$ to be a subset of the actual configuration space of the robot so that global coordinates can be defined, i.e., such that $\mathcal{Q}_{R}$ is embeddable in $\mathbb{R}^{n}$, or more simply $\mathcal{Q}_{R} \subset \mathbb{R}^{n}$. Calculating the mass and inertia properties of each link of the robot, coupled with the EulerLagrange equations $[23,29]$, yields the affine control systems $\left(f_{R}, g_{R}\right)$ :

$$
f_{R}(\theta, \dot{\theta})=\left[\begin{array}{c}
\dot{\theta} \\
-D^{-1}(\theta) C(\theta, \dot{\theta})
\end{array}\right], \quad g_{R}(\theta)=\left[\begin{array}{c}
\mathbf{0} \\
D^{-1}(\theta) B
\end{array}\right],
$$

where $D$ is the mass-inertia matrix, $C$ contains the Coriolis/centrifugal effects and gravitational terms, and $B \in \mathbb{R}^{n \times n}$ is the actuation matrix and assumed to be nonsingular, i.e., there is one independent actuator for each degree of freedom. Such robot models are said to be fully actuated. Finally, since we are assuming full-actuation, the set of admissible values is given by $U_{R} \subseteq \mathbb{R}^{n}$.

Domain and Guard: The domain specifies the allowable configuration of the system as specified by a unilateral constraint function $h$; for the bipeds considered in this paper, this function specifies that the non-stance foot must be above the ground, i.e., $h$ is the height of the non-stance foot and the system is subject to the unilateral constraint $h \geq 0$. Therefore, the domain $\mathcal{D}_{R}$ is given by:

$$
\mathcal{D}_{R}=\left\{(\theta, \dot{\theta}) \in T \mathcal{Q}_{R}: h(\theta) \geq 0\right\},
$$

where $T \mathcal{Q}_{R}$ is the tangent bundle of $\mathcal{Q}_{R}$. The guard is just the boundary of the domain with the additional assumption that the unilateral constraint is decreasing:

$$
S_{R}=\left\{(\theta, \dot{\theta}) \in T \mathcal{Q}_{R}: h(\theta)=0 \text { and } d h(\theta) \dot{\theta}<0\right\},
$$

where $d h(\theta)$ is the Jacobian of $h$ at $\theta$. 


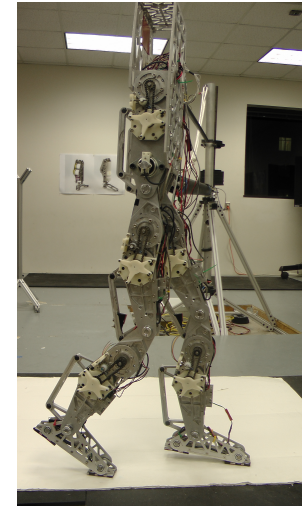

(a) Sideview of bipedal robot AMBER 3.

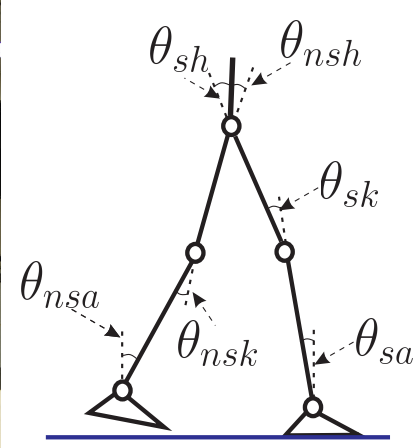

(b) Joint angles.

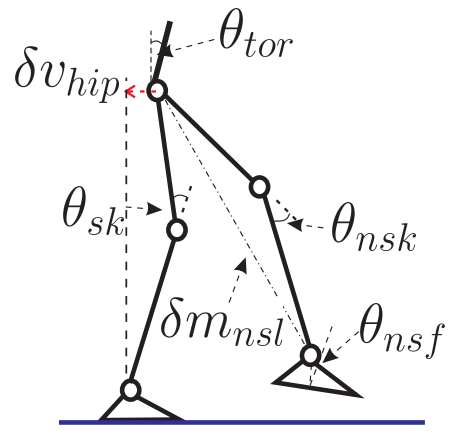

(c) Outputs.

Figure 3: The bipedal robot AMBER 3 (a), the joint angles (b) and outputs (c).

Discrete Dynamics. The discrete dynamics of the robot determine how the velocities of the robot change when the foot impacts the ground, while simultaneously swapping the roles of the "stance" and "non-stance" legs. In particular, the reset map $\Delta_{R}$ is given by:

$$
\Delta_{R}: S_{R} \rightarrow \mathcal{D}_{R}, \quad \Delta_{R}(\theta, \dot{\theta})=\left[\begin{array}{c}
\Delta_{\theta} \theta \\
\Delta_{\dot{\theta}}(\theta) \dot{\theta}
\end{array}\right],
$$

where $\Delta_{\theta}$ is the relabeling of the configuration variable associated with the stance and non-stance leg at impact. Here, $\Delta_{\dot{\theta}}$ determines the change in velocity due to impact (see $[16,14]$ for a detailed discussion).

Example 1. The model for the bipedal robot AMBER 3 considered in this paper is a special case of (2), with the parameters, e.g., masses, lengths and inertias, determined from a high-fidelity SolidWorks model (see Table 1). In particular, AMBER 3 is a 2D \%-link bipedal robot with feet (additional details regarding the development of the model can be found in Section 5). For this initial study, a simplified flat-footed gait will be assumed, resulting in a 6 degree of freedom 12-state model. The coordinates of $\mathcal{Q}_{R} \subset \mathbb{R}^{6}$ are denoted by $\theta=\left(\theta_{s a}, \theta_{s k}, \theta_{s h}, \theta_{n s h}, \theta_{n s k}, \theta_{n s a}\right)^{T}$ where, as illustrated in Fig. 3b, $\theta_{s a}$ is the angle of the stance ankle, $\theta_{\text {sk }}$ is the angle of the stance knee, $\theta_{\text {sh }}$ is the angle of the torso with the stance thigh, $\theta_{n s h}$ is the angle of the non-stance thigh with the torso, and $\theta_{n s k}$ is the angle of the non-stance (or swing) knee, and $\theta_{n s a}$ is the angle of the non-stance ankle (or foot). Since AMBER 3 is fully actuated, based upon this choice of coordinates $B=I_{6} \in \mathbb{R}^{6 \times 6}$. Note that we will take $U_{R}=\mathbb{R}^{6}$ for AMBER 3, and impose physical constraints that will restrict the admissible inputs to a subset of $U_{R}$ as will be discussed at the end of this section.

Walking Gaits. Before discussing the design specifications to be met through formal methods, it is first necessary to single out a class of desired solutions 


\begin{tabular}{|c|c|c|c|c|}
\hline \multicolumn{5}{|c|}{ Model Parameters } \\
\hline Part & $\begin{array}{c}\text { Mass } \\
(\mathrm{kg})\end{array}$ & $\begin{array}{c}\text { Length } \\
(\mathrm{mm})\end{array}$ & $\begin{array}{c}\text { Inertia } x \text {-axis } \\
\left(\mathrm{kgmm}^{2}\right)\end{array}$ & $\begin{array}{c}\text { Inertia } z \text {-axis } \\
\left(\mathrm{kgmm}^{2}\right)\end{array}$ \\
\hline Calf & 3.865 & 406.4 & 29032.44 & 3199.56 \\
\hline Thigh & 3.865 & 406.4 & 29032.44 & 3199.56 \\
\hline Torso & 10.418 & $251.1^{1}$ & 188576.35 & 59483.66 \\
\hline Foot & 1.151 & 152.4 & 1541.04 & 8498.06 \\
\hline
\end{tabular}

Table 1: Hardware parameters for AMBER 3.

to be realized on the hybrid system $\mathscr{H} \mathscr{C}_{R}$. In previous work of the authors, the focus was on asymptotically stable periodic walking motions [35, 4, 2], and solutions constructed through the composition of periodic motions. One reason that previous work was limited to periodic solutions was that classical nonlinear design tools are well adapted for characterizing various kinds of stable equilibrium points or periodic orbits of a closed-loop system, but are less well suited for characterizing classes of bounded trajectories with less structure that may also correspond to walking gaits. One of the take-home messages of this paper is that the marriage of classical nonlinear control techniques and formal methods is able to treat a larger class of trajectories.

Suppose now that a feedback controller $u(\theta, \dot{\theta})$ is applied to (2) with the end result being a hybrid system $\mathscr{H}_{R}$. Let $\chi^{\mathscr{H}_{R}}\left(\theta_{0}, \dot{\theta}_{0}\right)$ be a solution to this system with initial condition $\left(\theta_{0}, \dot{\theta}_{0}\right)$ and $c_{i}(t)=\left(\theta_{i}(t), \dot{\theta}_{i}(t)\right)$. In addition, let $p_{\mathrm{com}}^{x}(\theta)$ be the horizontal position of the center of mass of the robot, $\dot{p}_{\mathrm{com}}^{x}(\theta, \dot{\theta})$ the velocity of the forward position of the center of mass, and $p_{\mathrm{com}}^{y}(\theta)$ be the vertical position of the center of mass. Then we formalize the following notion of a walking gait for a bipedal robot. ${ }^{2}$

Definition 1. A solution $\chi^{\mathscr{H}_{R}}\left(\theta^{-}, \dot{\theta}^{-}\right)$to $\mathscr{H}_{R}$ is a walking gait if $\left(\theta^{-}, \dot{\theta}^{-}\right) \in$ $S_{R}$ and there exist ${ }^{3}$ constants $\tau_{\min }>0$ and $p_{\mathrm{com}}^{\min }>0$ such that

$$
\begin{aligned}
\tau_{i+1}-\tau_{i} & >\tau_{\text {min }} & \text { (Dwell Time) } \\
\dot{p}_{\text {com }}^{x}\left(\theta_{i}\left(\tau_{i+1}\right), \dot{\theta}_{i}\left(\tau_{i+1}\right)\right) & >0 & \text { (Progress) } \\
\min _{t \in I_{i}} p_{\text {com }}^{y}\left(\theta_{i}(t)\right) & >p_{\text {com }}^{\min } & \text { (Upright) }
\end{aligned}
$$

for all $i \in \mathbb{N}$. A walking gait is stable if for all $\gamma>0$ there exists a $\delta>0$ such that all solutions $\chi^{\mathscr{H}_{R}}\left(\theta_{0}, \dot{\theta}_{0}\right)$ with $\left(\theta_{0}, \dot{\theta}_{0}\right) \in B_{\delta}\left(\theta^{-}, \dot{\theta}^{-}\right) \cap S_{R}$ satisfy:

$$
\left(\theta_{i}\left(\tau_{i+1}\right), \dot{\theta}_{i}\left(\tau_{i+1}\right)\right) \in B_{\gamma}\left(\theta^{-}, \dot{\theta}^{-}\right) \cap S_{R}
$$

and are walking gaits.

\footnotetext{
${ }^{2}$ We define a ball of radius $\delta>0$ around a point $x$ by $B_{\delta}(x)=\left\{y \in \mathcal{D}_{R}:\|x-y\|<\delta\right\}$.

${ }^{3}$ These constants are defined by the user based upon the desired behavior of the walking gait.
} 


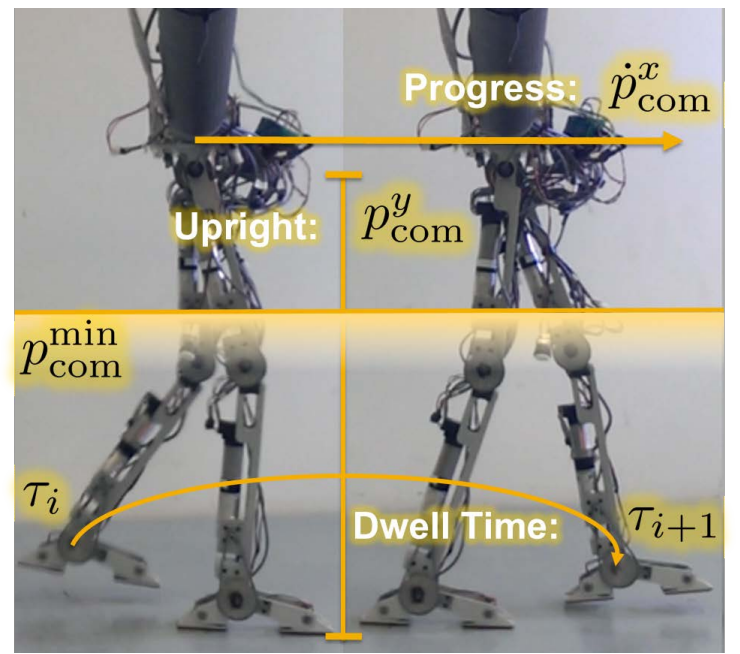

(a) Graphical illustration of the definition of walking.

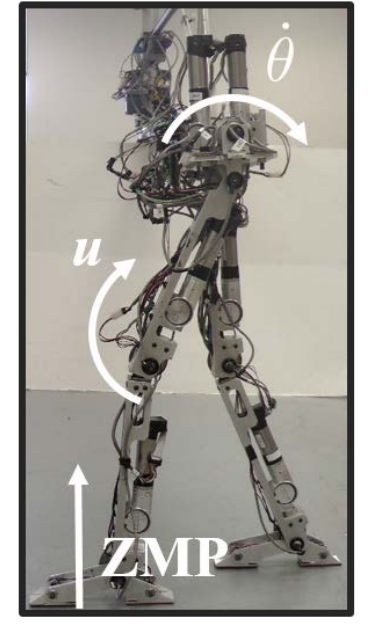

(b) Physical constraints on the robot.

Figure 4: Graphical illustration of both the elements that define walking (a) and the physical constraints on the robot (b).

The conditions singled out in Def. 1 are not universal prescriptions for walking. Rather, as mentioned previously, they are used to single out desirable features in a walking gait without restricting ourselves to periodic locomotion. In this case, the conditions ensure that there are no instantaneous transitions (e.g., no Zeno behavior [18]), guarantee that the center of mass makes forward progress via a velocity condition, and ensure that the robot is sufficiently upright (as defined by the user through $p_{\mathrm{com}}^{\min }$ ). Finally, note that due to the fact that we are only considering infinite solutions, inherent in this definition is the notion that the walking gait is indefinite.

Physical Specifications. The following physical specifications are required of a walking gait $\chi^{\mathscr{H}_{R}}$ :

Torque Bounds: From the definition of a hybrid control system, any input belonging to $U_{R}$ is allowed, yet we explicitly indicate the performance limitations of actuators through an additional physical constraint on a walking gait given by:

$$
\sup _{t \in I_{i}, i \in \mathbb{N}}\left\|u\left(\theta_{i}(t), \dot{\theta}_{i}(t)\right)\right\|_{\infty}<u_{\max }
$$

where $u$ is the control law that generated the gait and $u_{\max }$ is the maximum joint torque achievable by all of the actuators (this is assumed to be a uniform number for the sake of simplicity).

Velocity Bounds: As with torque bounds, we require that the maximum joint 
velocity stays under a maximum value, $\dot{\theta}_{\max }$, as specified by the actuators. In particular:

$$
\sup _{t \in I_{i}, i \in \mathbb{N}}\left\|\dot{\theta}_{i}(t)\right\|_{\infty}<\dot{\theta}_{\max }
$$

$Z M P$ Constraints $^{4}$ : We require that the feet remain flat during a walking gait, which results in zero moment point (ZMP) type constraints $[33,14]$. If $\eta_{s t}(\theta)$ is the position and orientation of the stance foot with respect to a fixed inertial frame, then ZMP conditions are determined by viewing $\eta_{s t}$ as a holonomic constraint. In particular, if $J_{\eta_{s t}}$ is the jacobian of $\eta_{s t}$, then the forces and moments at the stance foot are given by:

$$
\begin{aligned}
& F_{s t}(\theta, \dot{\theta}, u)= \\
& \left(J_{\eta_{s t}}(\theta) D(\theta)^{-1} J_{\eta_{s t}}(\theta)^{T}\right)^{-1}\left(J_{\eta_{s t}}(\theta) D(\theta)^{-1}(C(\theta, \dot{\theta})-B u)-\dot{J}_{\eta_{s t}}(\theta, \dot{\theta}) \dot{\theta}\right),
\end{aligned}
$$

where $F_{s t} \in \mathbb{R}^{3}$ for $2 \mathrm{D}$ walking robots and $F_{s t} \in \mathbb{R}^{6}$ for $3 \mathrm{D}$ walking robots. Conditions so that the foot does not roll during walking can be expressed through inequality constraints of the form:

$$
\sup _{t \in I_{i}, i \in \mathbb{N}} A_{\mathrm{ZMP}} F_{s t}\left(\theta_{i}(t), \dot{\theta}_{i}(t), u\left(\theta_{i}(t), \dot{\theta}_{i}(t)\right)\right)<0
$$

where $A_{\mathrm{ZMP}} \in \mathbb{R}^{2 \times 3}$ for $2 \mathrm{D}$ walking robots and $A_{\mathrm{ZMP}} \in \mathbb{R}^{4 \times 6}$ for $3 \mathrm{D}$ walking robots is a matrix that depends on the physical parameters of the feet of the robot (in particular, the length and the width of the foot [14]).

Foot Height Constraints: To achieve walking gaits on physical bipedal robots, it is important that the swing foot does not "scuff" before the end of a step. We introduce, therefore, foot scuffing constraints. Let $h(\theta)$ be the height (y position) of the non-stance (swing) foot, then we require that:

$$
\tau_{i+1}=\inf \left\{t>\tau_{i}: h\left(\theta_{i}(t)\right)=0\right\} .
$$

Note that this condition prevents the case when the foot "scuffs" the ground $(h(\theta)=0)$ before reaching the guard (where $\dot{h}(\theta, \dot{\theta})<0)$. As a result, this is a stronger condition that the one imposed on solutions (and, specifically, (ii)). We also note that such a $\tau_{i+1}$ must exist due to the dwell time condition in Definition 1.

Definition 2. A walking gait $\chi^{\mathscr{H}_{R}}$ is physically realizable if it satisfies constraints $(\mathrm{C} 1)-(\mathrm{C} 4)$.

Example 2. For AMBER 3, based upon the specifications of its actuators coupled with appropriate factors of safety, $u_{\max }=60 \mathrm{Nm}$ and $\dot{\theta}_{\max }=5 \mathrm{rad} / \mathrm{s}$. For

\footnotetext{
${ }^{4}$ Note that we could also consider friction conditions in a similar fashion but will omit doing so for brevity.
} 
the $Z M P$ constraints, $F_{s t}=\left(F_{s t}^{f x}, F_{s t}^{f y}, F_{s t}^{m z}\right)$ (see [14]) since AMBER 3 is a $2 D$ robot, and the ZMP constraints are determined by:

$$
A_{\mathrm{ZMP}}=\left[\begin{array}{ccc}
0 & -l_{h} & -1 \\
0 & -l_{t} & 1
\end{array}\right],
$$

where $l_{t}$ and $l_{h}$ are the length to the toe from the ankle and heel from the ankle, respectively.

\section{Dimension Reduction through Control}

This section builds on an existing nonlinear feedback control method to reduce the dimension of the robot model for which a correct-by-construction controller can be practically synthesized. The reduction technique utilizes the notion of a virtual constraint $[2,14,35]$. In particular, we will consider relative degree 2 outputs for which a classical nonlinear controller exists that drives these outputs to zero. Nulling the outputs will restrict the full-order dynamics of the robot to a low-dimensional surface - termed the partial zero dynamics - wherein the evolution of the system may be further dictated by relative degree 1 outputs. Through pre-feedback control laws, the dynamics of the relative degree 1 outputs defines a 2-dimensional linear hybrid control system. Solutions of this reducedorder hybrid system will be proven to yield solutions to the full-order dynamics. To accomplish this "lifting" of solutions from the reduced-order system to the full-order model, two technical extensions to results in $[2,14,35]$ are necessary to allow the study of the aperiodic solutions identified in Def. 1 in a formally correct manner. These will be noted as they are developed.

Virtual Constraints. Consider virtual constraints (or outputs) of the following form $[2,14]$ :

$$
\begin{aligned}
y_{1}(\theta, \dot{\theta}, v) & =y_{a, 1}(\theta, \dot{\theta})-v \\
y_{2}(\theta, \alpha) & =y_{a, 2}(\theta)-y_{d, 2}(\rho(\theta), \alpha),
\end{aligned}
$$

where $y_{1}$ and $y_{2}$ will be chosen so that they are relative degree 1 and (vector) relative degree 2 , respectively. In this case, $y_{a, 1}(\theta, \dot{\theta})$ is the "actual" velocitybased output and $v$ is the "desired" velocity. In the following, we will view $v$ as the control input to the system (after pre-feedback), and we synthesize $v$ through formal methods. Similarly, $y_{a, 2}(\theta)$ is the actual vector of outputs that modulate the posture of the robot and $y_{d, 2}(\rho(\theta), \alpha)$ gives the desired evolution of the associated configuration variables as dictated by parameters $\alpha$ in the desired evolution of the virtual constraints and a parameterization of time $\rho(\theta)$.

For the sake of simplicity, we will assume that the virtual constraints have a linear structure, namely

$$
\begin{aligned}
y_{a, 1}(\theta, \dot{\theta}) & =c \dot{\theta} \\
y_{a, 2}(\theta) & =H \theta
\end{aligned} \quad \text { s.t. } \quad \operatorname{rank}\left(\left[\begin{array}{c}
c \\
H
\end{array}\right]\right)=n
$$


and that $\rho(\theta)=c \theta-c \theta^{+}$, where $\theta^{+}$is the configuration at the beginning of a step, and will be specified later. The goal is to drive both the velocity and posture modulating outputs to zero, i.e., $y_{1} \rightarrow 0$ and $y_{2} \rightarrow 0$.

Example 3. In the case of $A M B E R$ 3, the virtual constraints considered in this paper are illustrated in Fig. 3c. In particular, as discussed in [21], ya,1 is the linearized velocity of the hip, $v$ is the desired velocity of the hip, $y_{a, 2}$ consists of a vector of configuration based functions and $y_{d, 2}$ is the time solution to a linear mass-spring-damper system parameterized by the linearized position of the hip.

Pre-Feedback Control. To set the stage for obtaining the reduced order dynamics that will be used to synthesize controllers for the system, we begin by applying a "pre-feedback" controller based upon feedback linearization [28] (note that this controller is a slight modification of the controller presented in $[2,3])$. With the goal of driving $y_{2} \rightarrow 0$ and shaping the dynamics of $y_{1}$ to be that of a linear system with control input $v$, consider the feedback controller:

$$
\begin{aligned}
u_{\mathrm{FB}}^{(\alpha, \varepsilon)}(\theta, \dot{\theta})=-\mathcal{A}^{-1}(\theta, \dot{\theta}) & \left(\left[\begin{array}{c}
0 \\
L_{f_{R}} L_{f_{R}} y_{2}(\theta)
\end{array}\right]\right. \\
+ & {\left.\left[\begin{array}{c}
L_{f_{R}} y_{a, 1}(\theta, \dot{\theta}) \\
2 \frac{1}{\varepsilon} L_{f_{R}} y_{2}(\theta, \dot{\theta}, \alpha)
\end{array}\right]+\left[\begin{array}{c}
\frac{1}{\varepsilon} y_{1}(\theta, \dot{\theta}) \\
\frac{1}{\varepsilon^{2}} y_{2}(\theta, \alpha)
\end{array}\right]\right), }
\end{aligned}
$$

with control gain $\varepsilon>0$ and decoupling matrix:

$$
\mathcal{A}(\theta, \dot{\theta})=\left[\begin{array}{c}
L_{g_{R}} y_{a, 1}(\theta, \dot{\theta}) \\
L_{g_{R}} L_{f_{R}} y_{2}(\theta, \dot{\theta}, \alpha)
\end{array}\right] .
$$

Here $L$ denotes the Lie derivative [28], and we assume that the decoupling matrix is invertible. It follows that $u_{\mathrm{FB}}^{(\alpha, \varepsilon)}(\theta, \dot{\theta})$ results in dynamics on the outputs given by:

$$
\begin{aligned}
\dot{y}_{a, 1} & =-\frac{1}{\varepsilon} y_{1} \\
\ddot{y}_{2} & =-2 \frac{1}{\varepsilon} \dot{y}_{2}-\frac{1}{\varepsilon^{2}} y_{2}
\end{aligned}
$$

and therefore, for a control gain $\varepsilon>0$, the control law $u_{\mathrm{FA}}^{(\alpha, \varepsilon)}$ renders the outputs exponentially stable [28]. That is, in the case when $v$ is a constant (and hence $\left.\dot{y}_{a, 1}=\dot{y}_{1}\right)$, the virtual constraints $y_{1} \rightarrow 0$ and $y_{2} \rightarrow 0$ exponentially at a rate of $\frac{1}{\varepsilon}$.

Partial Hybrid Zero Dynamics. While the introduced controller drives $y_{1} \rightarrow 0$ and $y_{2} \rightarrow 0$, we want to be able to modulate the relative 1 degree output (through $v$ ), while forcing the relative 2 degree output to remain zero and hence form an invariant surface. This motivates the introduction of the partial zero dynamics surface [2]:

$$
\mathbf{P} \mathbf{Z}_{\alpha}=\left\{(\theta, \dot{\theta}) \in T \mathcal{Q}_{R}: y_{2}(\theta, \alpha)=0, L_{f_{R}} y_{2}(\theta, \dot{\theta}, \alpha)=0\right\}
$$


We say that the hybrid system (2) has partial hybrid zero dynamics (PHZD) ${ }^{5}$ if:

$$
\Delta_{R}\left(S_{R} \cap \mathbf{P} \mathbf{Z}_{\alpha}\right) \subset \mathbf{P} \mathbf{Z}_{\alpha} .
$$

In particular, we can choose the parameters $\alpha$ so that this holds through an optimization of the form:

$$
\begin{aligned}
\alpha^{*}=\underset{\alpha \in \mathbb{R}^{5(n-1)}}{\operatorname{argmin}} & \operatorname{Cost}_{\mathrm{HD}}(\alpha) \\
\text { s.t. } & \Delta_{R}\left(S_{R} \cap \mathbf{P} \mathbf{Z}_{\alpha}\right) \subset \mathbf{P} \mathbf{Z}_{\alpha} .
\end{aligned}
$$

Note that the cost can be chosen based upon the specific objective of interest, i.e., minimizing the cost of transport, and that this optimization can be stated only in terms of the parameters $\alpha$ through the constructions introduced in [2].

The notion of PHZD allows for the construction of a hybrid system model for the reduced order dynamics defined by the surface (15). In particular, we reformulate the constructions in [35] in a way applicable to full-actuation [2]. Because of the specific form of $y_{a, 1}$ in (10) due to the linear output assumption, we begin by picking the following coordinates for the partial zero dynamics surface:

$$
\begin{aligned}
& z_{1}=c \theta \\
& z_{2}=y_{a, 1}(\theta, \dot{\theta})=c \dot{\theta}
\end{aligned}
$$

where $c \in \mathbb{R}^{1 \times n}$ as introduced in (10). As a result of the fact that we have full actuation and have completely linearized the dynamics with (11), the relative degree 1 output (8) evolves according to (13). Therefore, the partial hybrid zero dynamics evolve according to the linear ODE:

$$
\begin{aligned}
& \dot{z}_{1}=z_{2} \\
& \dot{z}_{2}=-\frac{1}{\varepsilon}\left(z_{2}-v\right) .
\end{aligned}
$$

where $v \in \mathbb{R}$ is viewed as a control input. The end result is, therefore, a linear control system:

$$
\dot{z}=A_{\mathbf{P Z}} z+B_{\mathbf{P Z}} v
$$

with $A_{\mathbf{P Z}}$ and $B_{\mathbf{P Z}}$ obtained from (19).

Impact Configurations. It is important to note that the proper choice of parameters $\alpha$ that determine the partial zero dynamics surface determine the configuration of the robot at impact (foot strike). In particular, the configuration of the robot at impact, $\theta^{-}$, is determined by the following requirement:

$$
\theta^{-}=\theta \quad \text { s.t. }\left[\begin{array}{c}
y_{2}(\theta, \alpha) \\
h(\theta)
\end{array}\right]=\left[\begin{array}{l}
0 \\
0
\end{array}\right] .
$$

\footnotetext{
${ }^{5}$ This formulation is based upon the notion of hybrid zero dynamics for underactuated bipedal robots [36].
} 
By virtue of the form of the relabeling matrix, $\Delta_{\theta}$, it follows that if $h\left(\theta^{-}\right)=0$ then $h\left(\theta^{+}\right)=0$ for $\theta^{+}=\Delta_{\theta} \theta^{-}$. Moreover, from the fact that $y_{2}(\theta, \alpha) \in \mathbb{R}^{n-1}$, for the proper choice of relative degree 2 outputs and parameters $\alpha,(21)$ has at least two solutions for $\theta \in \mathcal{Q}_{R}{ }^{6}$. Since the additional solutions are points where the foot scuffs the ground, i.e., points where $\dot{h} \geq 0$, we make the following assumption:

Assumption 1. Let $\alpha$ be parameters solving the optimization problem (16) and therefore guarantee (PHZD). Furthermore, assume that $\theta^{-}$and $\theta^{+}=\Delta_{\theta} \theta^{-}$are the only two points in $\mathcal{Q}_{R}$ satisfying (21).

Reduced Order Hybrid Dynamics. The advantage of the partial zero dynamics representation is that it yields a reduced-order hybrid system representation that dictates the behavior of the full order dynamics of the system. We will explicitly construct this hybrid system, and establish properties of its solutions relative to solutions of the full-order hybrid system.

Pick, once and for all, parameters $\alpha$ solving the optimization problem (16) and a point $\theta^{-}$satisfying Assumption 1 with $\theta^{+}=\Delta_{\theta} \theta^{-}$. We can therefore compute $z_{1}^{-}=c \theta^{-}$and $z_{1}^{+}=c \theta^{+}$. From this, since (PHZD) is satisfied, the discrete change in $z_{1}$ and $z_{2}$ can be determined via $[2,36]$ :

$$
\begin{aligned}
& z_{1}^{+}=c \Delta_{\theta} \theta^{-} \\
& z_{2}^{+}=\Delta_{\mathbf{P Z}}\left(\theta^{-}\right) z_{2}^{-}
\end{aligned}
$$

where $\theta^{-}$is a point that is chosen a priori and

$$
\Delta_{\mathbf{P Z}}\left(\theta^{-}\right):=c \Delta_{\dot{\theta}}\left(\theta^{-}\right) \Psi_{\mathbf{P Z}}\left(c \theta^{-}\right) .
$$

This defines a linear 2-dimensional hybrid control system:

$$
\mathscr{H} \mathscr{C}_{\mathbf{P Z}}=\left(\mathcal{D}_{\mathbf{P Z}}, U_{\mathbf{P Z}}, S_{\mathbf{P Z}}, \Delta_{\mathbf{P Z}}, f_{\mathbf{P Z}}, g_{\mathbf{P Z}}\right) .
$$

where the domain and guard are given by:

$$
\begin{aligned}
\mathcal{D}_{\mathbf{P Z}} & =\left\{z \in \mathbb{R}^{2}: z_{1}^{+} \leq z_{1} \leq z_{1}^{-}\right\}, \\
S_{\mathbf{P Z}} & =\left\{z \in \mathbb{R}^{2}: z_{1}=z_{1}^{-}\right\} .
\end{aligned}
$$

We will not (initially) restrict the control input $v$ and, therefore, $U_{\mathbf{P Z}}=\mathbb{R}$. The reset map, $\Delta_{\mathbf{P Z}}$, is a linear transformation as given by (22). Finally, the control system has $f_{\mathbf{P Z}}(z)=A_{\mathbf{P Z}} z$ and $g_{\mathbf{P Z}}(z)=B_{\mathbf{P Z}}$.

PHZD Reconstruction. We can use the relationship between the reduced order PHZD and the full-order dynamics, as afforded by the feedback control law, to reconstruct the full-order state of the system. Note that since $z_{1}$ is directly

\footnotetext{
${ }^{6}$ Note that, due to the trigonometric functions that yield $h$, it may be necessary to consider subsets of the configuration space $\mathcal{Q}_{R}$ that limit the number of solutions to (21).
} 
related to the parameterization of time, we can write $y_{d, 2}\left(z_{1}\right)=y_{d, 2}(\rho(\theta), \alpha)$ wherein it was assumed that we are working with a fixed parameter set $\alpha$. Therefore, defining

$$
\begin{aligned}
& \Phi_{\mathbf{P Z}}\left(z_{1}\right)=\left[\begin{array}{c}
c \\
H
\end{array}\right]^{-1}\left(\begin{array}{c}
z_{1} \\
y_{d, 2}\left(z_{1}\right)
\end{array}\right) \\
& \Psi_{\mathbf{P Z}}\left(z_{1}\right)=\left[\begin{array}{c}
c \\
H
\end{array}\right]^{-1}\left(\begin{array}{c}
1 \\
\frac{\partial y_{d, 2}\left(z_{1}\right)}{\partial z_{1}}
\end{array}\right)
\end{aligned}
$$

it follows that:

$$
\begin{aligned}
& \vartheta_{r}(z):=\Phi_{\mathbf{P Z}}\left(z_{1}\right) \\
& \dot{\vartheta}_{r}(z):=\Psi_{\mathbf{P Z}}\left(z_{1}\right) z_{2}
\end{aligned} \quad \Rightarrow\left(\vartheta_{r}(z), \dot{\vartheta}_{r}(z)\right) \in \mathbf{P} \mathbf{Z}_{\alpha}
$$

with $z=\left(z_{1}, z_{2}\right)^{T}$. Note that if we pick coordinates $\eta=\left(y_{2}, \dot{y}_{2}\right)$, since $y_{2}$ is a relative degree 2 output it follows that there is a diffeomorphism $\Pi:(\theta, \dot{\theta}) \rightarrow$ $(\eta, z)$. We also note that there is the canonical embedding $\iota_{\mathbf{P Z}}: \mathcal{D}_{\mathbf{P Z}} \rightarrow \mathcal{D}_{R}$ given by $\iota_{\mathbf{P Z}}(z)=\Pi^{-1}(0, z)$.

Key Properties. The entire purpose of the reduction step is prepare the ground for correct-by-construction synthesis on the basis of a model of a size that is amenable to existing algorithms. To support this process, it must be shown that correctness of the reduced-order closed-loop system induces correct behavior for the full-order system in an appropriate neighborhood of the invariant surface. This is established next.

Suppose that we have a feedback control law $v(z)$ that is applied to the hybrid control system $\mathscr{H}_{\mathscr{C}} \mathbf{P Z}$ with the end result being a hybrid system $\mathscr{H}_{\mathbf{P Z}}$. The application of this control law in (11) via $y_{1}$ (which depends on $v(z)$ ) to $\mathscr{H}_{\mathscr{C}_{R}}$, yields a hybrid system $\mathscr{H}_{R}$.

Theorem 1. Let $\chi^{\mathscr{H}_{\mathbf{P Z}}}=(\mathcal{I}, \mathcal{Z})$, with $\mathcal{Z}=\left\{z_{i}\right\}_{i \in \mathbb{N}}$, be a solution to $\mathscr{H}_{\mathbf{P Z}}$ with $\tau_{i+1}-\tau_{i} \geq \tau_{\min }$. If $\chi^{\mathscr{H}_{R}}=\left(\mathcal{I}, \mathcal{C}_{r}\right)$, with $\mathcal{C}_{r}=\left\{c_{i}^{r}\right\}_{i \in \mathbb{N}}$ where $c_{i}^{r}(t)=$ $\left(\vartheta_{r}\left(z_{i}(t)\right), \dot{\vartheta}_{r}\left(z_{i}(t)\right)\right)$, satisfies (Progress), (Upright), and (C1)-(C3), then $\chi^{\mathscr{H}_{R}}$ is a physically realizable walking gait of $\mathscr{H}_{R}$.

Proof. We need only verify that if $\chi^{\mathscr{H}_{\mathrm{PZ}}}$ is a solution to $\mathscr{H}_{\mathbf{P Z}}$ then $\chi^{\mathscr{H}_{R}}$ is a solution to $\mathscr{H}_{R}$; the remaining statements then follow from the fact that (Progress), (Upright), and (C1)-(C3) are satisfied for the reconstructed solution: $c_{i}^{r}(t)=\left(\vartheta_{r}\left(z_{i}(t)\right), \dot{\vartheta}_{r}\left(z_{i}(t)\right)\right)$. To establish that $\chi^{\mathscr{H}_{R}}$ is a solution to $\mathscr{H}_{R}$, we must verify both the continuous and discrete conditions on a solution to a hybrid system.

The continuous conditions on $\chi^{\mathscr{H}_{R}}$ are given by the requirement that $\dot{c}_{i}^{r}(t)=$ $f_{\mathrm{cl}}\left(c_{i}^{r}(t)\right)$, where $f_{\mathrm{cl}}$ is the closed-loop dynamics obtained by applying $v(z)$ to (3) via (19) and (11). Since the initial condition $\left(\vartheta_{r}\left(z_{0}(0)\right), \dot{\vartheta}_{r}\left(z_{0}(0)\right)\right) \in \mathbf{P} \mathbf{Z}_{\alpha}$, and because the dynamics in the $\eta$ and $z$ coordinates evolve in a decoupled fashion according to (13) and (14), we need only verify that $y_{2}\left(\vartheta_{r}\left(z_{i}(t)\right)\right)=0$ and $\dot{y}_{2}\left(\vartheta_{r}\left(z_{i}(t)\right), \dot{\vartheta}_{r}\left(z_{i}(t)\right)\right)=0$ for all $t \in I_{i}$ and $i \in \mathbb{N}$. This follows from the construction of $\vartheta_{r}$ and $\dot{\vartheta}_{r}$ and, specifically, (27) and (28). 
The discrete conditions on $\chi^{\mathscr{H}_{R}}$ are given by (i), (ii) and (iii) as stated in Section 2. Condition (i) is satisfied by Assumption 1 (which also implies (C4)), i.e., the boundary of the domain $\mathcal{D}_{R}$ cannot be reached until the guard $S_{R}$ is reached, and the configuration in which the guard is reached is given by $\theta^{-}$. Similarly, (ii) is satisfied again because the first configuration where the guard is reached is $\theta^{-}, z_{1}^{-}=c \theta^{-}$and $\tau_{i+1}$ satisfies (ii) for $\chi^{\mathscr{H}_{\mathbf{P Z}}}$. Finally, (iii) is satisfied again through the formulation of the reset map (23).

The previous result concerned executions of the closed-loop hybrid model where the initial conditions lie in the invariant surface defined by the partial zero dynamics. The next result strengthens the conclusions of Theorem 1 to address executions with initial conditions drawn from an open neighborhood of the invariant surface. This is clearly important for the applicability of the method. The results will also be used in Section 4 to establish an attractivity property for the behavior designed through formal methods.

To establish this extension, we utilize a notion of distance between a set and solutions. In particular, given a set $S$ and an execution $\chi^{\mathscr{H}_{R}}$ :

$$
\operatorname{dist}\left(\chi^{\mathscr{H}_{R}}, S\right)=\sup _{i \in \mathbb{N}} \inf _{t \in I_{i}, x \in S}\left\|c_{i}(t)-x\right\| .
$$

Theorem 2. Let $P \subset \mathcal{D}_{\mathbf{P Z}}$ be an invariant set of $\mathscr{H} \mathscr{C}_{\mathbf{P Z}}$ under a feedback control law $v(z)$. Then $\iota_{\mathbf{P Z}}(P) \subset \mathbf{P} \mathbf{Z}_{\alpha}$, and for any $\gamma>0$ there exists a $\delta>0$ such that for $\left\|\eta_{0}\right\|<\delta$ any walking gait $\chi^{\mathscr{H}_{R}}\left(\eta_{0}, z_{0}\right)$ with $z_{0} \in P$ satisfies:

$$
\operatorname{dist}\left(\chi^{\mathscr{H}_{R}}, \iota_{\mathbf{P Z}}(P)\right)<\gamma .
$$

Proof. Define $\kappa(i, t)$ such that

$$
\kappa(i, t)=\inf _{x \in \iota_{\mathbf{P Z}}(P)}\left\|c_{i}(t)-x\right\|
$$

for arbitrary $i \in \mathbb{N}$ and $t \in I_{i}$. Moreover, since $\iota_{\mathbf{P Z}}$ is the canonical embedding $\iota_{\mathbf{P Z}}(z)=\Pi^{-1}(0, z)$ and because the dynamics of the system evolve in a decoupled fashion according to (13) and (14), it follows that the only nontrivial component of the distance will be contributions from the $y_{2}$ and $\dot{y}_{2}$ dynamics. Formally, writing $\left(\eta_{i}(t), z_{i}(t)\right)=\Pi\left(c_{i}(t)\right)=\Pi\left(\theta_{i}(t), \dot{\theta}_{i}(t)\right)$, it follows that

$$
\begin{aligned}
\kappa(i, t) & \leq\left\|\eta_{i}(t)\right\|=\left\|\begin{array}{l}
y_{2}^{i}(t) \\
\dot{y}_{2}^{i}(t)
\end{array}\right\| \\
& \leq \underbrace{\frac{\lambda_{1}}{\varepsilon} e^{-\frac{\lambda_{2}}{\varepsilon} \tau_{\min }}}_{\beta(\varepsilon)}\left\|\begin{array}{l}
y_{2}^{i}\left(\tau_{i}\right) \\
\dot{y}_{2}^{i}\left(\tau_{i}\right)
\end{array}\right\|
\end{aligned}
$$

for some constants $\lambda_{1}, \lambda_{2}>0$, where the second inequality follows from [4] since $y_{2}$ and $\dot{y}_{2}$ evolve according to the linear system (14), picking $t=\tau_{i+1}$, and utilizing the dwell time assumption: $\tau_{i+1}-\tau_{i}>\tau_{\min }$.

To understand the role of the discrete dynamics, we note that in the coordinates $(\eta, z)$ we can decompose the reset map as follows: $\Delta_{R} \circ \Pi^{-1}(\eta, z)=$ 
$\left(\Delta_{\eta}(\eta, z), \Delta_{z}(\eta, z)\right)$. The assumption of partial hybrid zero dynamics (PHZD) implies that $\Delta_{\eta}(0, z)=0$. By assumption $\Delta_{\eta}$ is Lipschitz continuous with Lipschitz constant $L_{\Delta_{\eta}}$, wherein:

$$
\left\|\Delta_{\eta}(\eta, z)\right\|=\left\|\Delta_{\eta}(\eta, z)-\Delta_{\eta}(0, z)\right\| \leq L_{\Delta_{\eta}}\|\eta\| .
$$

Combining this with (30) and (31) implies that:

$$
\kappa(i, t) \leq \beta(\varepsilon)^{i+1} L_{\Delta_{\eta}}^{i} \delta \forall t \in I_{i}
$$

for any $i \in \mathbb{N}$. The RHS is a geometric sequence in $i$ with ratio $r(\varepsilon)=\beta(\varepsilon) L_{\Delta_{\eta}}$. Since $\beta(\varepsilon) \rightarrow 0$ as $\varepsilon \rightarrow 0$, there exists an $\bar{\varepsilon}>0$ such that $\beta(\varepsilon)<\frac{1}{L_{\Delta_{\eta}}}$, i.e. such that $r(\varepsilon)<1$, for all $0<\varepsilon<\bar{\varepsilon}$. Thus, for all $0<\varepsilon<\bar{\varepsilon}$ we have that

$$
\begin{aligned}
\operatorname{dist}\left(\chi^{\mathscr{H}_{R}}, \iota_{\mathbf{P Z}}(P)\right) & =\sup _{i \in \mathbb{N}} \inf _{t \in I_{i}} \kappa(i, t) \\
& \leq \sup _{i \in \mathbb{N}} r(\varepsilon)^{i} \beta(\varepsilon) \delta \\
& =\beta(\varepsilon) \delta \\
& <\frac{\delta}{L_{\Delta_{\eta}}}
\end{aligned}
$$

Therefore, picking $\delta<\gamma L_{\Delta_{\eta}}$ yields the desired result.

\section{Abstraction Based Controller Synthesis}

In this section, we show how to synthesize an abstraction based controller for the linear hybrid system $\mathscr{H} \mathscr{C}_{\mathbf{P Z}}$, defined in (24), enforcing the desired specifications by construction. The techniques to be employed are described in [37] (see also [31] for an introduction to abstraction based controller synthesis) and were developed for discrete-time systems. Hence, we start by defining discrete-time executions for a hybrid system $\mathscr{H}$.

Let $t_{s} \in \mathbb{R}^{+}$be a sampling time and let $\chi^{\mathscr{H}}=(\mathcal{I}, \mathcal{C})$ be a hybrid execution of hybrid system $\mathscr{H}$. A discrete-time hybrid execution of $\mathscr{H}$ with sampling time $t_{s}$, denoted by $\chi_{d}^{\mathscr{H}}=\left(\mathcal{I}_{d}, \mathcal{C}_{d}\right)$, is given by a collection of time intervals $\mathcal{I}_{d}=\left\{I_{d, i}\right\}_{i \in \mathbb{N}}$ where $I_{d, i}=\left\{\tau_{i}, \tau_{i}+t_{s}, \tau_{i}+2 t_{s}, \ldots, \tau_{i+1}\right\}$, and by a collection of functions $\mathcal{C}_{d}=\left\{c_{d, i}\right\}_{i \in \mathbb{N}}$ where each $c_{d, i}: I_{d, i} \rightarrow \mathcal{D}$ is given by the restriction of $c_{i}$ to the set $I_{d, i}$.

The starting point for abstraction based controller synthesis is the construction of a finite-state abstraction $S\left(\mathscr{H} \mathscr{C}_{\mathbf{P Z}}\right)$ of $\mathscr{H} \mathscr{C}_{\mathbf{P Z}}$ by following the methods in [37]. This abstraction comes equipped with an $\varepsilon$-approximate alternating simulation relation from $S\left(\mathscr{H} \mathscr{C}_{\mathbf{P Z}}\right)$ to $\mathscr{H} \mathscr{C}_{\mathbf{P Z}}$ guaranteeing that any controller synthesized for $S\left(\mathscr{H} \mathscr{C}_{\mathbf{P Z}}\right)$ can be refined to a controller for $\mathscr{H} \mathscr{C}_{\mathbf{P Z}}$ resulting in the same closed-loop behavior up to an error of $\varepsilon \in \mathbb{R}^{+}$. In other words, let us denote by $S\left(\mathscr{H}_{\mathbf{P Z}}\right)$ the hybrid system resulting from composing $S\left(\mathscr{H} \mathscr{C}_{\mathbf{P Z}}\right)$ with a controller and let us denote by $\mathscr{H}_{\mathbf{P Z}}$ the hybrid system resulting from 
composing $\mathscr{H} \mathscr{C}_{\mathbf{P Z}}$ with the refined controller. Then, for every discrete-time hybrid execution $\chi_{d}^{S\left(\mathscr{H}_{\mathbf{P Z}}\right)}$ any corresponding discrete-time hybrid execution $\chi_{d}^{\mathscr{H}_{\mathbf{P Z}}}$ satisfies:

$$
\operatorname{dist}\left(\chi_{d}^{S\left(\mathscr{H}_{\mathbf{P Z}}\right)}, \chi_{d}^{\mathscr{H}_{\mathbf{P Z}}}\right) \leq \varepsilon
$$

Moreover, $\varepsilon$ is a design parameter that can be made as small as desired, at the expense of a larger finite-state abstraction.

Convexity of Reachable Sets. The key technical assumption required for the results in [37] is the possibility of computing an over-approximation of the reachable set of $\mathscr{H} \mathscr{C}_{\mathbf{P z}}$. Hence, we describe in this section how this can be efficiently done. We start by recalling a few notions.

A vector $x \in \mathbb{R}^{n}$ is a convex combination of $m$ vectors $x_{1}, \ldots, x_{m} \in \mathbb{R}^{n}$ if $x$ can be written as

$$
x=\sum_{i=1}^{m} \lambda_{i} x_{i}, \quad \lambda_{i} \geq 0, \quad \sum_{i=1}^{m} \lambda_{i}=1 .
$$

A set is convex if it contains the convex combination of its elements. A point $x \in B$ is called an extreme point of a compact convex set $B \subset \mathbb{R}^{n}$ if it cannot be represented by a convex combination of any two points $x_{1}, x_{2} \in B$, with $x_{1} \neq x$ and $x_{2} \neq x$. The convex hull of a set $B \subseteq \mathbb{R}^{n}$ is the set of all convex combinations of points in $B$ and is denoted $\operatorname{conv}(B)$. It follows that any compact convex set is the convex hull of its extreme points.

Definition 3. The set reached by the trajectories of (19) from $B \subseteq \mathcal{D}_{\mathbf{P Z}}$ in time $t_{s} \in \mathbb{R}_{0}^{+}$under constant input $v$ is denoted by $\mathcal{R}_{v}^{t_{s}}(B)$ and defined as:

$$
\mathcal{R}_{v}^{t_{s}}(B)=\left\{z^{\prime} \in \mathbb{R}^{2} \mid z(0) \in B \wedge z\left(t_{s}\right)=z^{\prime}\right\}
$$

where $z(t)$ is a solution to (19) with the constant input $v$ and initial condition $z(0)$. Moreover, we define $\mathcal{R}_{v}(B)$ by:

$$
\mathcal{R}_{v}(B)=\cup_{t_{s} \in \mathbb{R}_{0}^{+}} \mathcal{R}_{v}^{t_{s}}(B)
$$

To simplify notation we write $\mathcal{R}_{v}(x)$ rather than $\mathcal{R}_{v}(\{x\})$ when $B$ is the singleton $\{x\}$.

We now show that the intersection of $\mathcal{R}_{v}(B)$ with the guard set $S_{\mathbf{P Z}}$ is a convex set.

Theorem 3. Consider the convex set $B \subseteq \mathbb{R}^{2}$ defined by:

$$
B=\left[a_{1}, b_{1}\right] \times\left[a_{2}, b_{2}\right]
$$

with $a_{1}, a_{2}, b_{1}, b_{2} \in \mathbb{R}, a_{1}<b_{1}$, and $a_{2}<b_{2}$. Denote by $\hat{z}_{1}, \ldots, \hat{z}_{4} \in B$ the extreme points of $B$. If the linear dynamics (19) satisfies $\dot{z}_{1} \geq c$ on $\mathcal{D}_{\mathbf{P z}}$ for some $c>0$, then:

$$
\mathcal{R}_{v}(B) \cap S_{\mathbf{P Z}}=\operatorname{conv}\left\{\mathcal{R}_{v}\left(\hat{z}_{1}\right) \cap S_{\mathbf{P Z}}, \ldots, \mathcal{R}_{v}\left(\hat{z}_{4}\right) \cap S_{\mathbf{P Z}}\right\}
$$


Proof. In this proof we denote by $F_{t}: \mathbb{R}^{2} \rightarrow \mathbb{R}^{2}$ flow of the linear system (19) with constant input $v$, i.e., if $z(t)$ is the solution of (19) with initial condition $z^{\prime}$ and constant input $v$ then $F_{t}\left(z^{\prime}\right)=z(t)$. We will also say that (19) is transversal to the boundary of $B$ at $z^{\prime} \in B$ when $\dot{z}_{1} \neq 0$ for $z^{\prime}$ belonging to the vertical boundaries and when $\dot{z}_{2} \neq 0$ for $z^{\prime}$ belonging to the horizontal boundaries.

We first state and prove two facts.

Fact 1: If $z$ belongs to the boundary of $\mathcal{R}_{v}(B) \cap S_{\mathbf{P Z}}$, then $z$ is the image under $F_{t}$ of a boundary point of $B$ for some $t \in \mathbb{R}$.

It suffices to show that $F_{t}$ maps interior points of $B$ to interior points of $\mathcal{R}_{v}(B) \cap S_{\mathbf{P Z}}$. But this follows directly from the fact that $F_{-t}$, the inverse of $F_{t}$, exists and is continuous. The inverse image of an open set by a continuous map is an open set. Hence, let $O^{\prime} \subseteq B$ be an open set containing a point $z^{\prime}$ in the interior of $B$ and let $t_{s}$ satisfy $F_{t_{s}}\left(z^{\prime}\right)=z \in S_{\mathbf{P Z}}$. Since $\dot{z}_{1} \geq c>0$ and the guard is given by $z_{1}=z_{1}^{-}, t_{s}$ exists. Then the set $O=F_{-t_{s}}\left(O^{\prime}\right)$ is an open set containing the point $z$ in $\mathcal{R}_{v}(B) \cap S_{\mathbf{P Z}}$. Since $O \subseteq \mathcal{R}_{v}(B)$ and $O \cap S_{\mathbf{P Z}}$ is open in the topology induced on $S_{\mathbf{P Z}}$ by the standard topology in $\mathbb{R}^{2}, z$ is an interior point of $\mathcal{R}_{v}(B) \cap S_{\mathbf{P Z}}$.

Fact 2: If $z$ belongs to the boundary of $\mathcal{R}_{v}(B) \cap S_{\mathbf{P Z}}$, then $z$ cannot be the image under $F_{t}$ of a point in the boundary of $B$ where (19) is transversal to the boundary.

We will show that if $z^{\prime}$ belongs to the boundary of $B$ and (19) is transversal to the boundary at $z^{\prime}$, then the flow takes $z^{\prime}$ to an interior point of $\mathcal{R}_{v}(B) \cap S_{\mathbf{P Z}}$. Let $O^{\prime}$ be an open set in the boundary of $B$ containing $z^{\prime}$. Consider the set $B^{\prime}=B \cup F_{t}\left(O^{\prime}\right)$ for sufficiently small $t$ ( $t$ is positive if the vector field points to the outside of $B$ and negative otherwise). It is clear that $\mathcal{R}_{v}(B)=\mathcal{R}_{v}\left(B^{\prime}\right)$. Moreover, we can now take an open (in $\mathbb{R}^{2}$ ) subset $O$ of $B^{\prime}$ containing $z^{\prime}$. Applying the argument used to prove Fact 1 , we see that $F_{t}$ takes $z^{\prime}$ into an interior point of $\mathcal{R}_{v}(B) \cap S_{\mathbf{P Z}}$.

Proof of Theorem 3: Facts 1 and 2 tell us that boundary points of $\mathcal{R}_{v}(B) \cap S_{\mathbf{P Z}}$ are the image under the flow of extreme points of $B$ or of boundary points of $B$ where (19) is not transversal. The assumption $\dot{z}_{1} \geq c>0$ implies that the transversality condition can only fail on the horizontal boundaries. Moreover, the dynamics of $z_{2}$ given by (19) shows that if (19) is not transversal at a point on a horizontal boundary then all the points in that horizontal boundary are on the same trajectory. We thus conclude that boundary points of $\mathcal{R}_{v}(B) \cap S_{\mathbf{P Z}}$ are the image under the flow of extreme points of $B$. Let now $\gamma:[0,1] \rightarrow \mathbb{R}^{2}$ be a continuous curve contained in $B$ and joining the extreme point $\hat{z}_{1}$ to the extreme point $\hat{z}_{2}$, i.e., $\gamma(0)=\hat{z}_{1}, \gamma(1)=\hat{z}_{2}$, and $\gamma(r) \in B$ for $r \in[0,1]$. By continuity of the map $F_{t} \circ \gamma$ we have $\mathcal{R}_{v}\left(\cup_{r \in[0,1]}\{\gamma(r)\}\right) \cap S_{\mathbf{P Z}}=\operatorname{conv}\left(\mathcal{R}_{v}\left(\hat{z}_{1}\right), \mathcal{R}_{v}\left(\hat{z}_{2}\right)\right)$. Since this argument does not depend on the choice of extreme points, the result follows.

The abstraction techniques in [37] require the over-approximation of $\mathcal{R}_{v}^{t_{s}}(B)$ when the guard is not reached in $t_{s}$ units of time. In this case the linearity of 
(19) implies that $\mathcal{R}_{v}^{t_{s}}(B)$ is a convex set and can be computed as:

$$
\operatorname{conv}\left(\mathcal{R}_{v}^{t_{s}}\left(\hat{z}_{1}\right), \ldots, \mathcal{R}_{v}^{t_{s}}\left(\hat{z}_{4}\right)\right) .
$$

If the guard can be reached in $t_{s}$ units of time or less, then we need to overapproximate the set of points that can be reached up to the time the guard is hit and immediately after the reset. This set can be over-approximated by:

$$
\begin{gathered}
\left(\operatorname{conv}\left(\mathcal{R}_{v}^{t_{s}}\left(\hat{z}_{1}\right), \ldots, \mathcal{R}_{v}^{t_{s}}\left(\hat{z}_{4}\right)\right) \cap \mathcal{D}_{\mathbf{P Z}}\right) \\
\cup \Delta_{\mathbf{P Z}}\left(\operatorname{conv}\left\{\mathcal{R}_{v}\left(\hat{z}_{1}\right) \cap S_{\mathbf{P Z}}, \ldots, \mathcal{R}_{v}\left(\hat{z}_{4}\right) \cap S_{\mathbf{P Z}}\right\}\right) .
\end{gathered}
$$

Once again, all the sets are convex and can thus be efficiently computed since we only have to perform numerical simulations for the vertices of $B$.

Walking Gait Generation. One of the main advantages of abstraction based control is the possibility to enforce the specifications by construction. In our case, for a stable robot walking gait, there are seven specifications that have to be satisfied: the (Dwell Time), (Progress) and (Upright) constraints in Definition 1 , as well as the physical requirements $(\mathrm{C} 1),(\mathrm{C} 2),(\mathrm{C} 3)$, and $(\mathrm{C} 4)$. For our system, the constraints (Dwell Time), (Upright), and (C4) are enforced by the choice of output functions (8),(9) and the feedback linearizing controller. The (Progress) constraint is automatically satisfied by the dynamics since $\dot{z}_{1}=z_{2}$ and $\mathcal{D}_{\mathbf{P Z}}$ only includes points where $z_{2}$ is strictly positive. Therefore, we only have to cater to the physical constraints (C1)-(C3).

We thus synthesize a controller forcing the closed-loop trajectories to remain in the set $P=P_{1} \cap P_{2} \cap P_{3}$ for all time where each set $P_{i}$ describes the constraint $(\mathrm{C} i)$ in the PHZD:

$$
\begin{aligned}
& P_{1}=\left\{z \in \mathcal{D}_{\mathbf{P Z}}:\left|u\left(\vartheta_{r}(z), \dot{\vartheta}_{r}(z)\right)\right|<u_{\max }\right\} \\
& P_{2}=\left\{z \in \mathcal{D}_{\mathbf{P Z}}:\left|\dot{\vartheta}_{r}(z)\right|<\dot{\theta}_{\max }\right\} \\
& P_{3}=\left\{z \in \mathcal{D}_{\mathbf{P Z}}: A_{\mathrm{ZMP}} F_{s t}\left(\vartheta_{r}(z), \dot{\vartheta}_{r}(z), u\left(\vartheta_{r}(z), \dot{\vartheta}_{r}(z)\right)\right)<0\right\} .
\end{aligned}
$$

Main Result. Recall that we denote by $\mathscr{H}_{\mathbf{P Z}}$ the hybrid system obtained by composing $\mathscr{H} \mathscr{C}_{\mathbf{P Z}}$ with the refined controller. This composition restricts the behavior of $\mathscr{H} \mathscr{C}_{\mathbf{P Z}}$ in two different ways: by restricting the available inputs, and by restricting the initial conditions. The set of initial conditions is denoted by $\mathcal{D}_{\mathbf{P Z}}^{\text {init }}$ and is a subset of $\mathcal{D}_{\mathbf{P Z}}$. It then follows from Proposition 9.4 in [31] that the hybrid executions of $\mathscr{H}_{\mathbf{P Z}}$ starting in $\mathcal{D}_{\mathbf{P Z}}^{\text {init }}$ remain in:

$$
P^{\varepsilon}=\left\{z \in \mathcal{D}_{\mathbf{P Z}} \mid\left\|z-z^{\prime}\right\| \leq \varepsilon \text { for some } z^{\prime} \in P\right\},
$$

for all time. By bounding the inter-sample behavior using a standard Lyapunovtype argument $[22,24,17]$ we conclude that the (continuous-time) hybrid executions of $\mathscr{H}_{\mathbf{P Z}}$ remain in $P^{\delta\left(\varepsilon, t_{s}\right)}$ for all time where $\delta$ is a continuous and increasing function of $\varepsilon$ and $t_{s}$ satisfying $\delta(\varepsilon, 0)=0$. Therefore, we can always find a subset $\bar{P}$ of $P$ and a choice of $t_{s}$ and $\varepsilon$ so that $\bar{P}^{\delta\left(\varepsilon, t_{s}\right)} \subseteq P$. By synthesizing a controller enforcing the stricter constraints defined by $\bar{P}$ we then guarantee that executions remain in $P$ as desired. This discussion, when coupled with Theorem 1 and 2, can be summarized in the following result. 
Theorem 4. Let $\mathscr{H}_{\mathbf{P Z}}$ be the hybrid system resulting from composing $\mathscr{H} \mathscr{C}_{\mathbf{P Z}}$ with the controller obtained by refining the controller synthesized for the finitestate abstraction of $\mathscr{H}_{\mathscr{C}} \mathbf{P Z}$. Any hybrid execution of $\mathscr{H}_{\mathbf{P Z}}$ starting in $\mathcal{D}_{\mathbf{P Z}}^{\text {init }}$ remains in $P$ for all time. Moreover, any hybrid execution of $\mathscr{H}_{R}$ with initial condition in $\iota_{\mathbf{P Z}}\left(\mathcal{D}_{\mathbf{P Z}}^{\text {init }}\right)$ is a physically realizable stable walking gait.

\section{Simulation and Experimental Results}

In this section, we present simulation and experimental results for the custom built bipedal robot AMBER 3. Additionally, we include information about how the abstraction building software PESSOA [27] is used to generate mappings for inputs to the zero dynamics. We present a brief description of how this mapping is refined to a feedback controller. The end result is the realization of this controller in simulation and the illustration that it is able to enforce constraints that were not enforceable by traditional gait generation software. The section concludes with experimental realization on AMBER 3; this sets the stage for a qualitative discussion in the next section.

To achieve the simulation results presented, we utilizes two control methods as a point of comparison: existing hybrid zero dynamics based controllers for fully actuated robots that have proven successful on hardware [34, 30, 15, 38], termed the "constant $v$ " controller, and the abstraction-based controllers presented in this paper. Importantly, the simulation results are performed to both show the improved performance of the abstraction-based controller in terms of realizing the specifications enumerated in the first part of this paper. In this context, the small gap between theory and simulation will be seen (due to discretization and numeric approximation). The simulation results set the stage for experimental realization on the robotic platform AMBER 3. Taking advantage of the significant gear ratio between the actuators and the links to approximate the inverse dynamics controller, i.e., we approximate the synthesized controllers with a time-based trajectory tracking implementation. Data from the experiments will be presented in this section. A qualitative discussion of our interpretation of the experiments will be presented in Sect. 6 so that fact and opinion are carefully separated. To the authors knowledge, the experimental trials presented here will be the first time that a "correct-by-construction" controller in any form, approximate or otherwise, has been implemented on a bipedal robot.

Control Synthesis. We consider two separate controllers: a nominal controller in which $v$ is held at a constant value and a controller in which $v$ is selected according to the abstraction-based technique outlined in Section 4. Both controllers were synthesized using the same set of parameters $\alpha$ found by solving (16) with the additional constraints $(\mathrm{C} 1)$ and $(\mathrm{C} 2)$. No feasible solution could be found that satisfied (C3) with constant $v$, so this constraint was relaxed.

The over-approximation of the reachable sets based on Theorem 3 has been implemented in the tool PESSOA, see [27]. In order to compute the finitestate abstraction we restricted the set of states to the operating region $Z^{\text {abstr }}=$ 

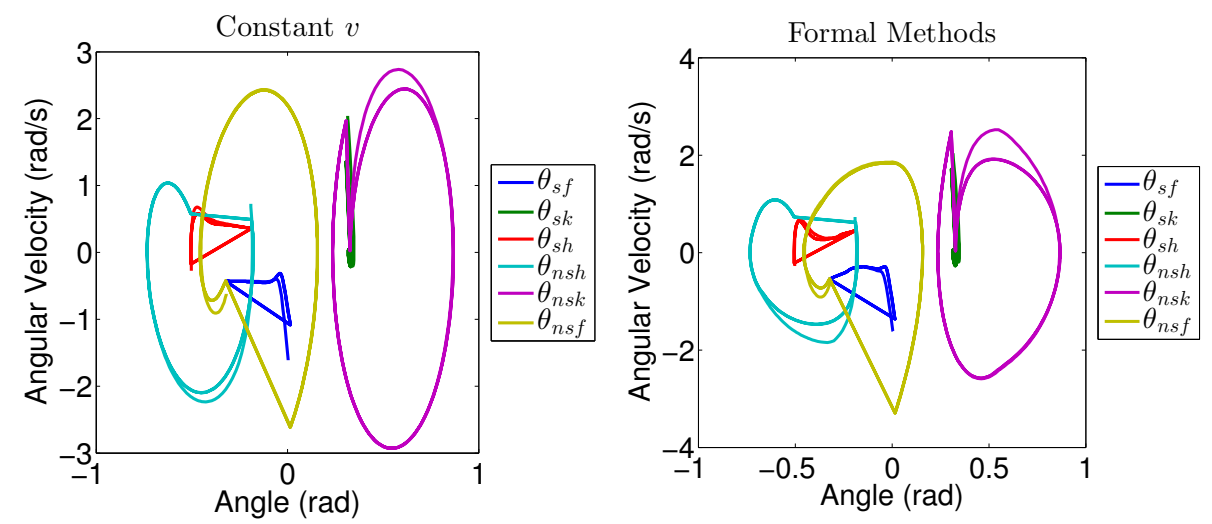

(a) Phase portrait for the state of the system $(\theta, \dot{\theta})$ over four steps. The maximum angular velocity, $\pm 5 \mathrm{rad} / \mathrm{s}$ (Constraint $(\mathrm{C} 1)$ ), was never violated by the system under either controller.
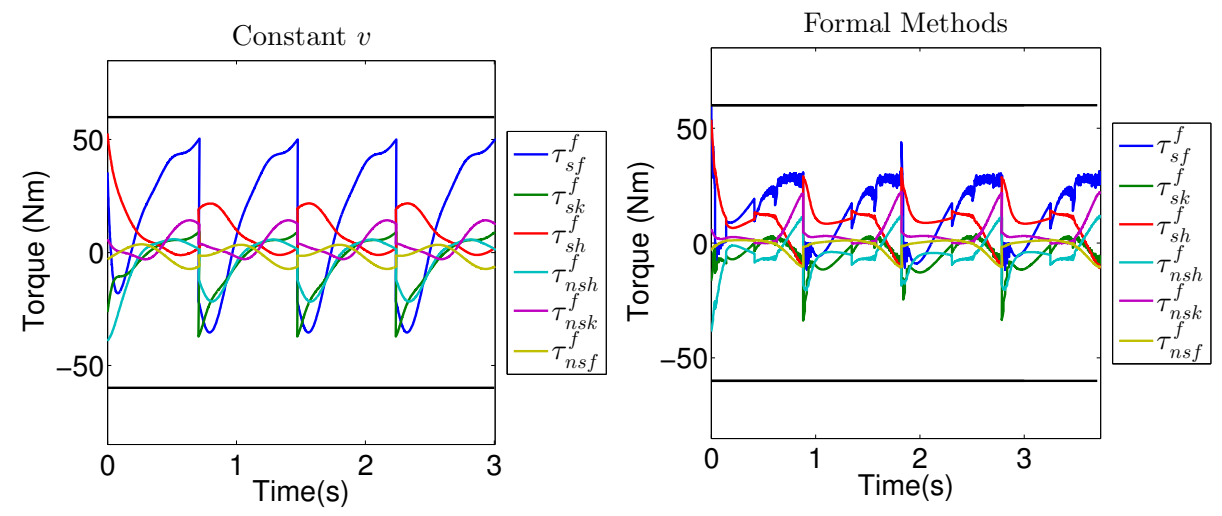

(b) The torques applied at each of the joints over four steps. The torque bounds of $\pm 60 \mathrm{Nm}$ are illustrated with horizontal black lines (Constraint (C2)). The torque remains bounded under each of the controllers.
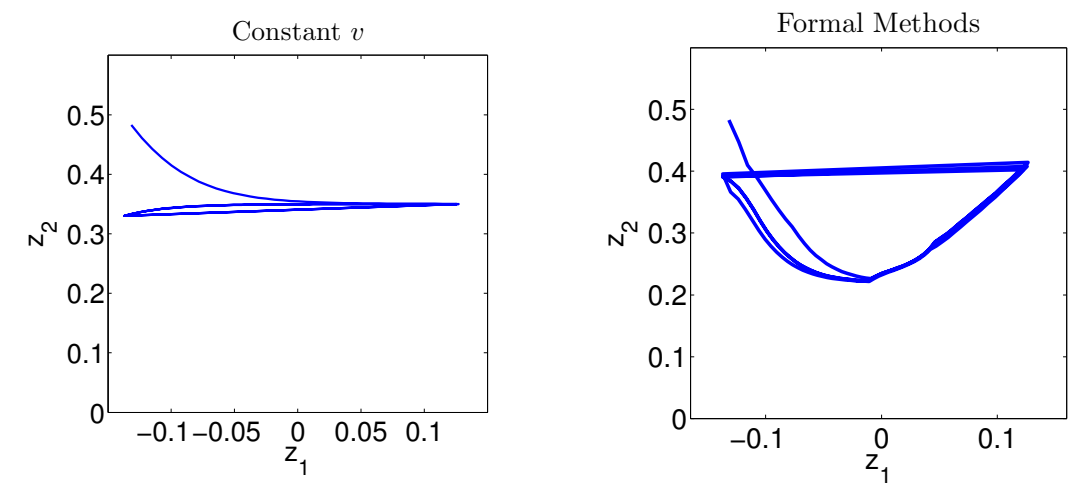

(c) Phase portrait for the zero dynamics coordinates $\left(z_{1}, z_{2}\right)$ over four steps. In both cases, the controller induces stable limit cycles in these coordinates

Figure 5: Simulations demonstrating different specifications for the walking gait obtained from a constant $v$ controller as generated in [21] and the formal methods controller. 

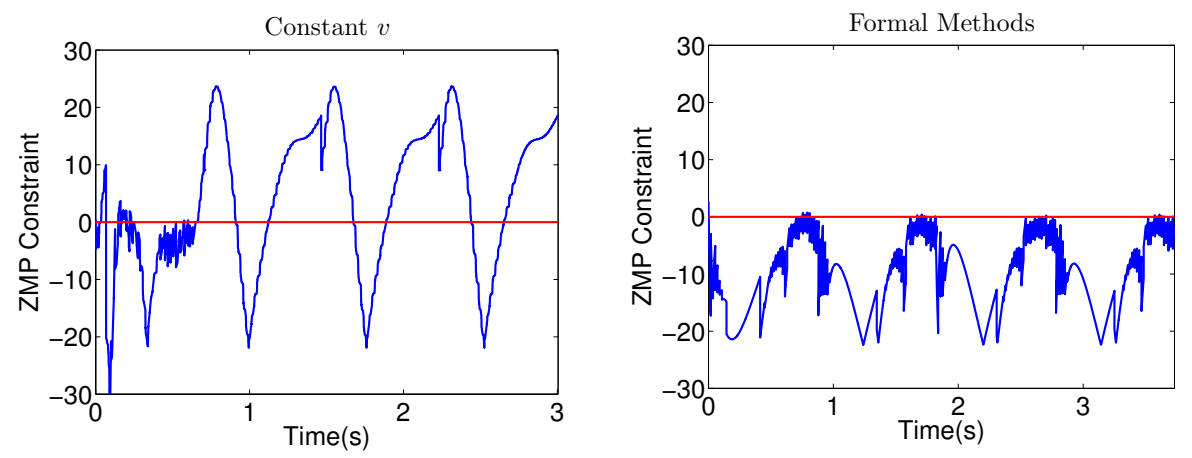

(a) Maximum violation of (C3) over four simulated steps. The upper bound of this constraint is equal to zero as illustrated by the horizontal red line. As we can see, the constant $v$ controller causes this constraint to be significantly violated, while the formal methods controller obeys this bound.
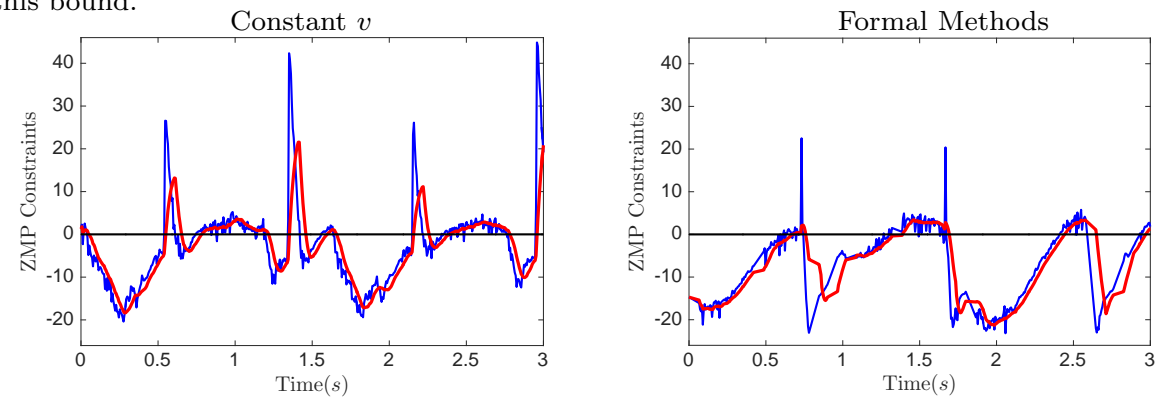

-Actual _ Moving Avg.

-Actual _ Moving Avg.

(b) Maximum violation of (C3) for constant $v$ controller (left) and the formal methods controller (right) calculated from experimental data. The upper bound of this constraint is equal to zero as illustrated by the horizontal black line. For both of the controllers, the constraint is frequently violated; however, the formal methods controller violates it to a lesser degree - this is evidenced by the moving average (in red). In particular, the constant $v$ controller has large spikes that violate the constraints, while the abstraction based controller only has small spikes (corresponding to foot strike) where the constraints is violated. The difference between these controllers is further illustrated in Fig. 11, wherein the actual ZMP point remains in the foot for a longer duration. See the discussion at the end of this section for further details.

Figure 6: The maximum value of the violation of Constraint (C3), i.e. $\left\|A_{\mathrm{ZMP}} F_{s t}\right\|_{\infty}$ for simulated and experimental data. 
$[-0.165,0.16] \times[0,0.6]$ and the input space to $U^{a b s t r}=[0.22,0.6]$. The operating region $Z^{\text {abstr }}$ contains $z_{1}^{-}$and $z_{1}^{+}$. The abstraction is then computed by dividing $Z^{\text {abstr }}$ into boxes $B$ of length 0.005 , by quantizing the input space $U^{\text {abstr }}$ with a resolution $\mu=0.0025$, and time discretization of $t_{s}=0.018 \mathrm{~s}$. See [37] for the definition of these parameters. The state space is covered by 7153 boxes and we consider 152 different input values. The abstraction was computed in about 3.5 hours on a computer with a $2.6 \mathrm{GHz}$ dual core processor. A controller for this abstraction that maintains the system within the set (36) was found after less than 5 seconds.

The controller synthesized by PESSOA is a mapping $\nu: Z^{a b s t r} \rightarrow 2^{Q_{\mu}\left(U^{a b s t r}\right)}$ such that $\nu(z)$ is the set of all quantized inputs that can be applied at state $z$ that can enforce safety ${ }^{7}$. This is illustrated in Fig. 7 , where the total number of available inputs, i.e. $|\nu(z)|$, is shown for each point $z \in Z^{\text {abstr }}$. In the state space of the abstraction, many states exist for which no input enforcing the specifications exists at all; however these states are not part of $\mathcal{D}_{\mathbf{P Z}}^{\text {init }}$ and are never reached. The non-deterministic controller is refined to a deterministic mapping

$$
v_{f m}(z)=\underset{v \in \nu(z)}{\arg \min } \operatorname{CoT}(z, v),
$$

where $C o T$ is the cost of transport. The input that is applied to the system, called here the "formal methods controller", is given as

$$
v(t)=v_{f m}\left(z\left(n(t) t_{s}\right)\right) \forall t \in\left[n(t) t_{s},(n(t)+1) t_{s}\right)
$$

where $n(t)$ is the maximum integer $n$ such that $n t_{s}<t$

Simulation Results. Simulation results comparing the constant $v$ controller and the formal methods controller are shown in Fig. 5. Fig. 5a and Fig. 5c show four steps of AMBER 3 in the $(\theta, \dot{\theta})$ plane, and in the $\left(z_{1}, z_{2}\right)$ plane, respectively. Note that for both the constant $v$ controller and the formal methods controller, when starting from some nearby point, the trajectory eventually settles into a stable periodic orbit. This behavior is by design in the constant $v$ controller, but this phenomenon occurs with the formal methods controller even though we did not include it in the safety specification. In Fig. 5c, we see that due to the varying $v, z_{2}$ changes much more in the case of the abstraction based controller than in the case of constant $v$. We also note that the constraint $(\mathrm{C} 2)$ is satisfied since the magnitude of the angular velocities never exceed $5 \mathrm{rad} / \mathrm{s}$. Fig. 5b and Fig. 6a show the satisfaction of the torque constraint and the ZMP constraint $(\mathrm{C} 1)$ and $(\mathrm{C} 3)$, respectively. The constant $v$ controller is not able to enforce the ZMP constraint, while the formal methods controller enforces all of the constraints. Together these simulation results indicate that the formal methods controller can be used to generate a stable, physically realizable walking

\footnotetext{
$\Delta$.

${ }^{7}$ Notation: $2^{A}$ is the power set of $A$ and $Q_{\Delta}(B)$ is the discretization of $B$ with resolution
} 


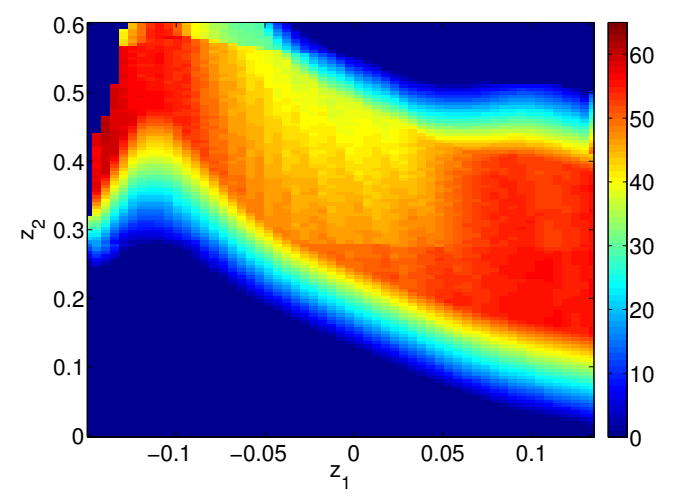

Figure 7: Number of available inputs for each state in the domain of the abstraction-based controller.

gait, and in particular, is able to generate such a gait when other methods are insufficient.

Experimental Platform and Methods. Both the constant $v$ and the approximated formal-methods controllers are implemented on the AMBER 3 robot, shown in Fig. 2 and Fig. 3a. The kinematic parameters of the robot are listed in Table 1. The main processing unit onboard the robot is a National Instruments C-Rio. The C-Rio communicates with six ELMO motion controllers through an EtherCAT network. Each controller powers a joint motor and reads data through an attached encoder. National Instruments modules read sensor input from the torso encoder and push-buttons located on the heels and toes. The software architecture used to control AMBER 3 is almost identical to the implementation previously used for AMBER 2 (described in [21]).

We approximated the two controllers by integrating equation (19) forward over time with the input $v^{*}=0.34$ for the constant $v$ controller and $v(t)$ for the formal methods controller. Then, we used the mapping (28) to reconstruct the trajectories in the full state space of the robot. The reconstructed trajectories were then tracked online via PD controllers at each joint ${ }^{8}$.

Experimental Results. One step of the walking gait generated by the formal method's controller is illustrated in the tiles shown in Fig. 8. A video comparing the two controllers can be found at [1]. Experimental data collected from the time-based implementation of each controller is shown in Figures 9. Figure 9a illustrates that each gait is stable and shows that the maximum angular velocity bound of $\pm 5 \mathrm{rad} / \mathrm{s}$ is respected. Similarly, Fig. $9 \mathrm{~b}$ shows that the torque applied to each joint in the robot is well within the bound of $\pm 60 \mathrm{Nm}$.

\footnotetext{
${ }^{8} \mathrm{PD}$ tracking was used instead of directly implementing the torque controllers because position and velocity measurements on the hardware have proven to be more reliable than torque measurements.
} 


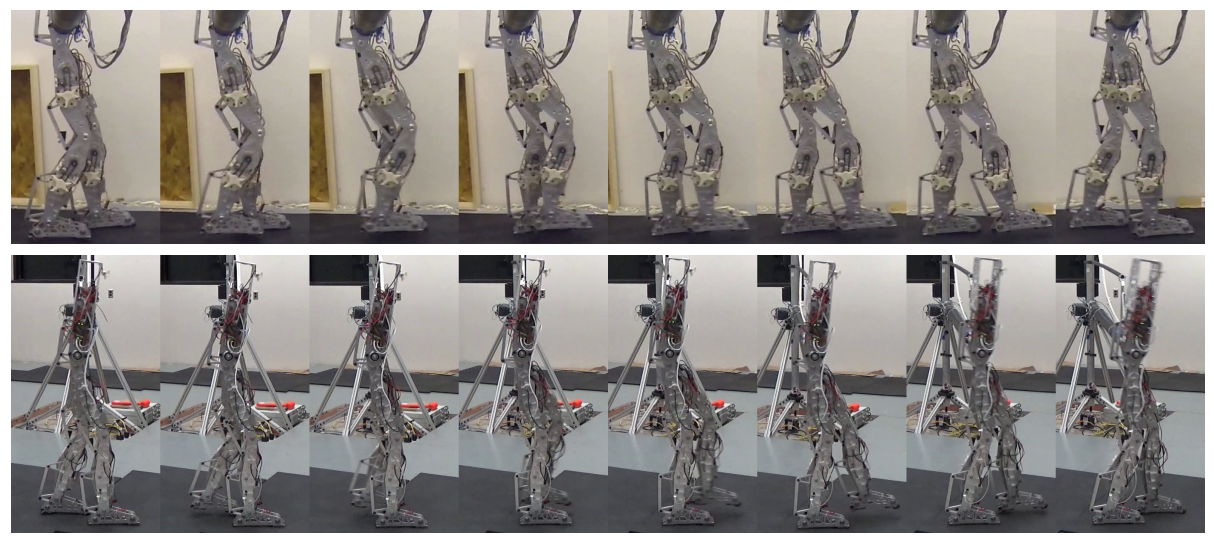

Figure 8: Tiles of the walking gait generated by the formal methods controller (video at [1].) The images in the top row are from the point of view of the boom. The images in the bottom row were taken from outside the radius of the boom.

The ZMP specification given by constraint (C3) is not directly measured by any sensor on the robot. Instead, the data presented in Fig. 10 is calculated from the experimentally collected position, velocity and torque at each of the robot's joints. A related quantity that gives insight into the ability of the controllers to keep the feet flat while walking (the reason for constraint (C3)) is the location of the Zero Moment Point (ZMP) along the foot, i.e. the point along the ground in which the net moment due to the torque acted on the robot and ground reaction forces is zero [7]. When this point is located inside the foot, the foot does not roll. On the other hand, if the ZMP is in front of the foot, the heel is lifted, and if it is located behind the foot, the toe is lifted. Figure 11 shows that the ZMP constraint is violated by each of the controllers.

\section{Qualitative Discussion}

In this section, we provide our personal interpretation of the experiments, all the while keeping in mind that any model will always be an approximation of reality, and hence even if we had "exactly" implemented the "formally correct controller", when placed in closed-loop with the robot, it would no longer be formally correct. The experimental data show that neither controller satisfied the ZMP specification. This said, as engineers, we should ask ourselves whether the experiments with the controller based on formal methods revealed any redeeming qualities ${ }^{9}$ that merit further exploration.

Figure 10 shows the portion of time that the ZMP remains within the foot is $0.4975,0.4835$ and 0.7902 for the constant $v$ trials, and $0.8352,0.7652$, and 0.7942 for the formal methods trials, respectively. In a professional design,

\footnotetext{
${ }^{9}$ The reviewers objected to our interpretations unless we stated them with these caveats.
} 

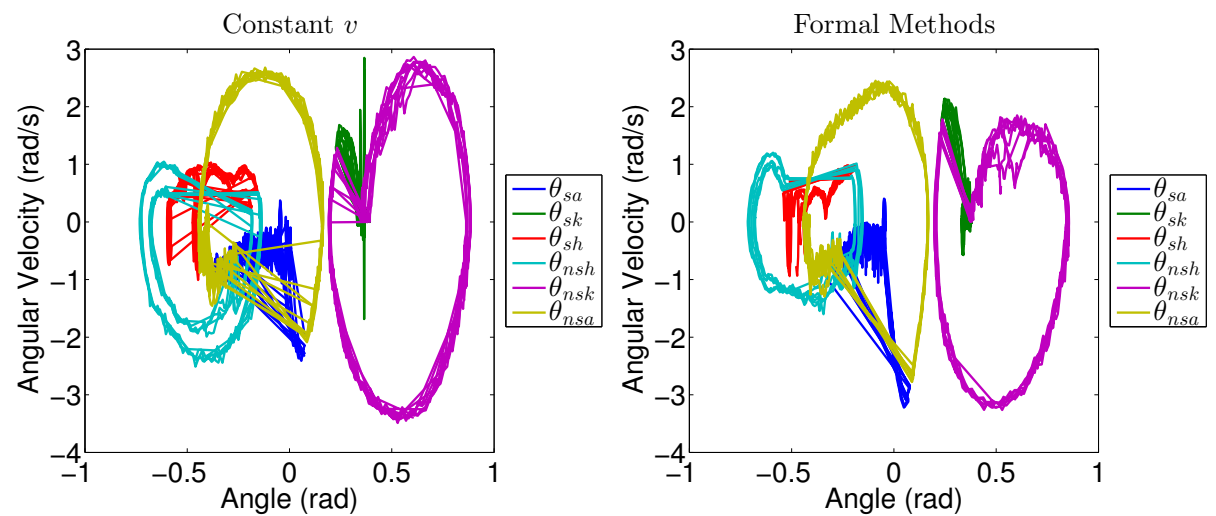

(a) Phase portrait for the state of the system $(\theta, \dot{\theta})$. The maximum allowed angular velocity, $\pm 5 \mathrm{rad} / \mathrm{s}$ (Constraint $(\mathrm{C} 1)$ ), was never violated by any joint in the robot under either controller.
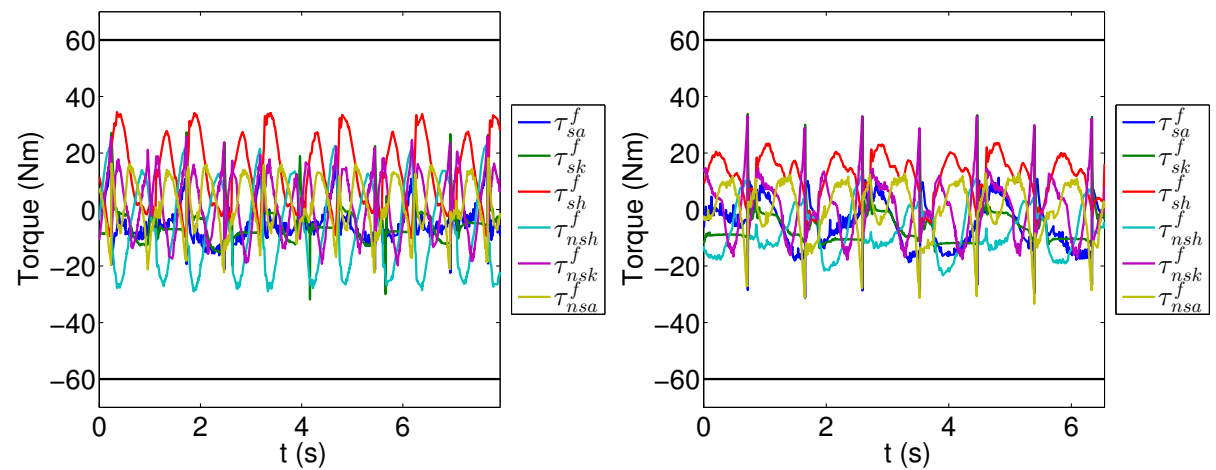

(b) The torques applied at each of the joints of the robots. The torque bounds of $\pm 60 \mathrm{Nm}$ are illustrated with horizontal black lines (Constraint $(\mathrm{C} 2)$ ). The torque remains bounded under each of the controllers.

Figure 9: Experimental data collected from walking trials of AMBER 3 under the constant $v$ controller (left) and the formal methods controller (right). 

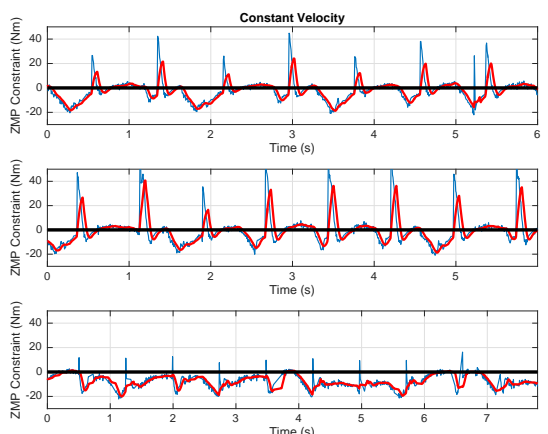
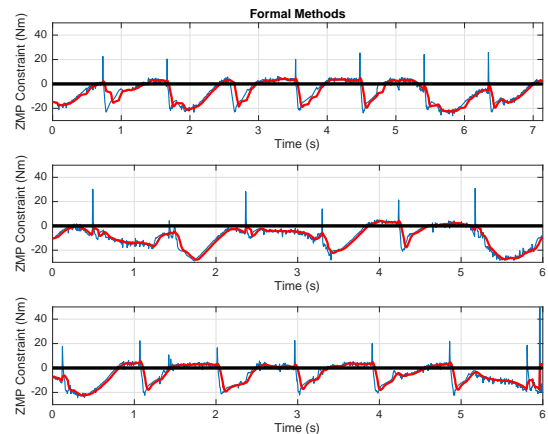

Figure 10: The violation of constraint (C3) for three experimental trials with the constant velocity controller and the formal methods controller; note that this is the same quantity plotted in Fig. 6 except it is not plotted over a much longer time window and over multiple experimental runs.
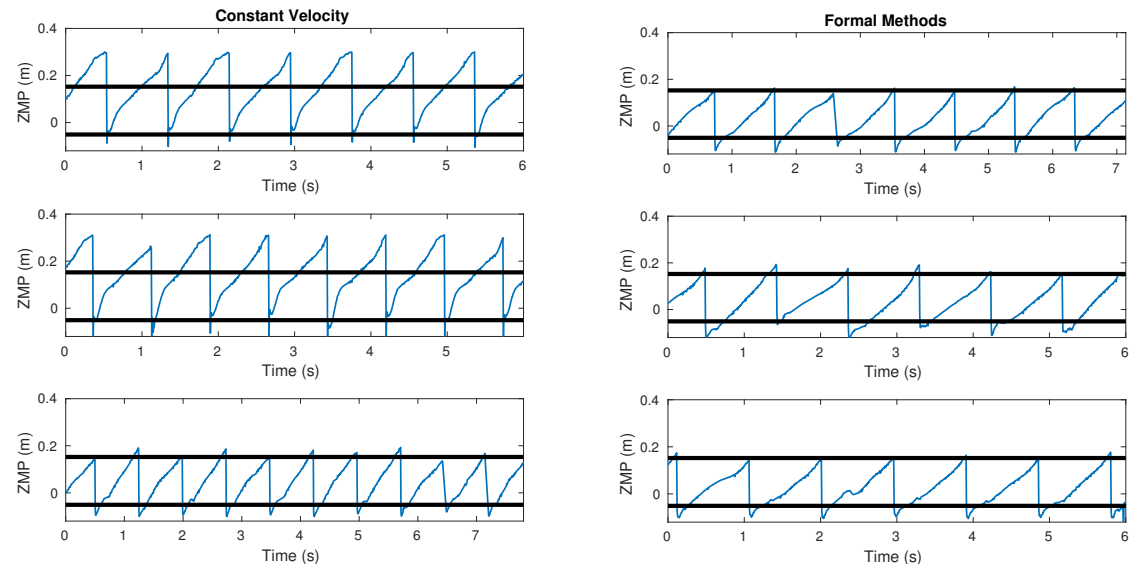

Figure 11: The location of the zero moment point relative to the point on the ground directly below the stance ankle. The black lines represent the boundaries of the foot; when the blue line indicating the instantaneous position of the ZMP leaves the region bounded by the black lines, a violation of the ZMP constraint occurs, allowing the foot to roll about its extremities. The portion of time the ZMP remains within the foot is $0.4975,0.4835$ and 0.7902 for the constant $v$ trials and $0.8352,0.7652$, and 0.7942 for the formal methods controller. 
margins would be included when formulating the specifications. Such practical precautions were not taken here because our goal was to qualitatively evaluate how a based on formal methods would perform on a real robot. Figure 10 reveals, nevertheless, that the inclusion of very small margins on the ZMP constraint would result in the formal method's controller meeting the specification. In our opinion, even though foot roll was not completely eliminated, the experiments indicate that the controller based on formal methods does a better job of controlling foot roll than the constant velocity controller.

We attribute the violation of the ZMP constraint to two factors: the difference between the designed pre-feedback control and the more reliable PD implementation on the physical hardware, and unmodeled phenomena such as imperfections in the walking surfaces and interactions with the boom. Possible ways to bridge these gaps include using counterexamples (i.e. data from failed experiments) in the synthesis algorithm, refining the synthesized controller using progressively more complex models, and "robustifying" the mapping of $v_{f m}$ by assigning inputs for states outside the safe region to drive the system back to safety, i.e. render the safe set invariant and attractive. In addition, as mentioned above, in a professional engineering implementation, margins would be included when doing the actual design.

\section{Conclusion}

In this paper, we showed how to combine the theory of virtual constraints with formal methods to generate correct-by-construction controllers for a highlydynamic non-linear system. The use of virtual constraints reduces the dimension of the planning space of the problem, while abstraction-based control synthesis allows for the synthesis of inputs in this low-dimensional space that enforce safety and hardware constraints to yield physically realizable walking. The main theoretical result of this paper indicates that this combination of methods yields a solution to the full-order system that satisfies given safety specifications. The practical relevance of the theoretical work was investigated by preforming the first experimental implementation of a controller based on formal methods on a bipedal walking robot. The experiments compared a formally correct controller and a heurtisc controller. In our qualitative comparison of the results, we argued that the controller based on formal methods was better able to satisfy important constraints on the robot's evolution, in particular, the zero moment point constraint, than a nominal gait found via optimization techniques. Future research directions include extending the technique in this paper to handle nonlinear zero dynamics and using abstractions to synthesize controllers for underactuated systems, e.g. bipedal robots with unactuated ankles.

\section{Acknowledgments}

This research is supported by NSF CPS Awards 1239055, 1239037 and 1239085. Experiments were performed at Texas A\&M University, College Station, Texas. The robot AMBER 3 was designed and built by Eric Ambrose. The authors would like to thank Aakar Mehra for his assistance with experiments. 
[1] AMBER-Lab. Abstraction-based control for AMBER 3.0. [Video file], Retrieved from http://youtu.be/UKuI7cEOEzs.

[2] A. D. Ames. Human-inspired control of bipedal walking robots. Automatic Control, IEEE Transactions on, 59(5):1115-1130, May 2014.

[3] A. D. Ames, E. A. Cousineau, and M. J. Powell. Dynamically stable bipedal robotic walking with $\mathrm{NAO}$ via human-inspired hybrid zero dynamics. In Proceedings of the 15th ACM international conference on Hybrid Systems: Computation and Control, pages 135-144. ACM, 2012.

[4] A. D. Ames, K. Galloway, K. Sreenath, and J. Grizzle. Rapidly exponentially stabilizing control Lyapunov functions and hybrid zero dynamics. Automatic Control, IEEE Transactions on, 59(4):876-891, 2014.

[5] A. D. Ames, P. Tabuada, B. Schürmann, W.-L. Ma, S. Kolathaya, M. Rungger, and J. W. Grizzle. First steps toward formal controller synthesis for bipedal robots. In Proceedings of the 18th International Conference on Hybrid Systems: Computation and Control, HSCC '15, pages 209-218, New York, NY, USA, 2015. ACM.

[6] S. A. Burden, H. Gonzalez, R. Vasudevan, R. Bajcsy, and S. S. Sastry. Metrization and Simulation of Controlled Hybrid Systems. IEEE Transactions on Automatic Control, 60(9):2307-2320, 2015.

[7] C. Chevallereau, D. Djoudi, and J. W. Grizzle. Stable bipedal walking with foot rotation through direct regulation of the zero moment point. IEEE TRO, 25(2):390-401, Apr. 2008.

[8] A. Colombo and D. Del Vecchio. Supervisory control of differentially flat systems based on abstraction. In 50th IEEE Conference on Decision and Control and European Control Conference., pages 6134-6139. IEEE, 2011.

[9] A. Colombo and A. Girard. An approximate abstraction approach to safety control of differentially flat systems. In European Control Conference, pages 4226-4231. IEEE, 2013.

[10] G. E. Fainekos, A. Girard, H. Kress-Gazit, and G. J. Pappas. Temporal logic motion planning for dynamic robots. Automatica, 45(2):343-352, Feb. 2009.

[11] J. Fu, H. G. Tanner, and J. Heinz. Concurrent multi-agent systems with temporal logic objectives: Game theoretic analysis and planning through negotiation. IET Control Theory and Applications, 9(3):465-474, 2015.

[12] R. Goebel, R. Sanfelice, and A. Teel. Hybrid dynamical systems. IEEE Contr. Syst. Mag., 29(2):28-93, Apr. 2009.

[13] J. W. Grizzle, G. Abba, and F. Plestan. Asymptotically stable walking for biped robots: Analysis via systems with impulse effects. IEEE TAC, 46(1):51-64, Jan. 2001. 
[14] J. W. Grizzle, C. Chevallereau, A. D. Ames, and R. W. Sinnet. 3D bipedal robotic walking: models, feedback control, and open problems. In 8th IFAC Symposium on Nonlinear Control Systems, 2010.

[15] A. Hereid, S. Kolathaya, M. S. Jones, J. Van Why, J. W. Hurst, and A. D. Ames. Dynamic multi-domain bipedal walking with ATRIAS through SLIP based human-inspired control. In Proceedings of the 17th international conference on Hybrid systems: computation and control, pages 263-272. ACM, 2014.

[16] Y. Hürmüzlü and D. B. Marghitu. Rigid body collions of planar kinematic chains with multiple contact points. Intl. J. of Robotics Research, 13(1):8292, Feb. 1994.

[17] C. M. Kellett, H. Shim, and A. R. Teel. Further results on robustness of (possibly discontinuous) sample and hold feedback. IEEE Transactions on Automatic Control, 49(7):1081-1089, Jan. 2004.

[18] A. Lamperski and A. Ames. Lyapunov theory for Zeno stability. Automatic Control, IEEE Transactions on, 58(1):100-112, Jan. 2013.

[19] J. Liu, N. Ozay, U. Topcu, and R. M. Murray. Synthesis of reactive switching protocols from temporal logic specifications. IEEE Transactions on Automatic Control, 58(7):1771-1785, July 2013.

[20] J. Lygeros, K. H. Johansson, S. N. Simic, J. Zhang, and S. S. Sastry. Dynamical properties of hybrid automata. Automatic Control, IEEE Transactions on, 48(1):2-17, 2003.

[21] W.-L. Ma, H.-H. Zhao, S. Kolathaya, and A. D. Ames. Human-inspired walking via unified PD and impedance control. In Robotics and Automation (ICRA), 2014 IEEE International Conference on, pages 5088-5094, May 2014.

[22] M. Mazo Jr, A. Anta, and P. Tabuada. An ISS self-triggered implementation of linear controllers. Automatica, 46(8):1310-1314, 2010.

[23] R. M. Murray, Z. Li, and S. S. Sastry. A Mathematical Introduction to Robotic Manipulation. CRC Press, Boca Raton, Mar. 1994.

[24] D. Nesic and A. R. Teel. Input-output stability properties of networked control systems. IEEE Transactions on Automatic Control, 49(10):16501667, Jan. 2004.

[25] H. Park, A. Ramezani, and J. W. Grizzle. A finite-state machine for accommodating unexpected large ground-height variations in bipedal robot walking. IEEE Trans. Robot., 29(2):331-345, 2013.

[26] H. Park, K. Sreenath, J. W. Hurst, and J. W. Grizzle. Identification of a bipedal robot with a compliant drivetrain: Parameter estimation for control design. IEEE Contr. Syst. Mag., 31(2):63-88, Apr. 2011. 
[27] P. Roy, P. Tabuada, and R. Majumdar. Pessoa 2.0: A controller synthesis tool for cyber-physical systems. In Proceedings of the 14 th International Conference on Hybrid Systems: Computation and Control, pages 315-316, 2011.

[28] S. S. Sastry. Nonlinear Systems: Analysis, Stability and Control. Springer, New York, June 1999.

[29] M. W. Spong, S. Hutchinson, and M. Vidyasagar. Robot modeling and control, volume 3. Wiley, 2006.

[30] K. Sreenath, H. Park, I. Poulakakis, and J. W. Grizzle. A compliant hybrid zero dynamics controller for stable, efficient and fast bipedal walking on MABEL. Intl. J. of Robotics Research, 30(9):1170-1193, 2011.

[31] P. Tabuada. Verification and Control of Hybrid Systems: A Symbolic Approach. Springer, 2009.

[32] A. Ulusoy, S. L. Smith, X. C. Ding, C. Belta, and D. Rus. Optimality and robustness in multi-robot path planning with temporal logic constraints. The International Journal of Robotics Research (IJRR), 32(8):889-911, July 2013.

[33] M. Vukobratović and B. Borovac. Zero-moment point- thirty five years of its life. International Journal of Humanoid Robotics, 1(01):157-173, 2004.

[34] E. R. Westervelt, G. Buche, and J. W. Grizzle. Experimental validation of a framework for the design of controllers that induce stable walking in planar bipeds. Intl. J. of Robotics Research, 24(6):559-582, 2004.

[35] E. R. Westervelt, J. W. Grizzle, C. Chevallereau, J. H. Choi, and B. Morris. Feedback Control of Dynamic Bipedal Robot Locomotion. CRC Press, Boca Raton, June 2007.

[36] E. R. Westervelt, J. W. Grizzle, and D. E. Koditschek. Hybrid zero dynamics of planar biped walkers. IEEE TAC, 48(1):42-56, 2003.

[37] M. Zamani, G. Pola, M. Mazo Jr., and P. Tabuada. Symbolic models for nonlinear control systems without stability assumptions. IEEE Transactions on Automatic Control, 57(7):1804-1809, July 2012.

[38] H.-H. Zhao, W.-L. Ma, M. Zeagler, and A. Ames. Human-inspired multicontact locomotion with AMBER2. In ACM/IEEE International Conference on Cyber-Physical Systems (ICCPS), pages 199-210, 2014. 